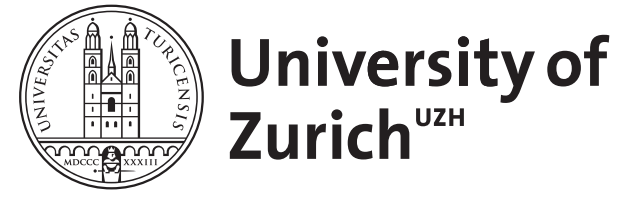

Zurich Open Repository and Archive

University of Zurich

University Library

Strickhofstrasse 39

CH-8057 Zurich

www.zora.uzh.ch

Year: 2009

\title{
Betriebsinterne Arbeitsmärkte, Hierarchie und Neueinstellungen: Eine empirische Untersuchung mit Personaldaten
}

\author{
Janssen, S ; Pfeifer, C
}

DOI: https://doi.org/10.1007/s11573-009-0243-z

Posted at the Zurich Open Repository and Archive, University of Zurich ZORA URL: https://doi.org/10.5167/uzh-23620

Journal Article

Published Version

Originally published at:

Janssen, S; Pfeifer, C (2009). Betriebsinterne Arbeitsmärkte, Hierarchie und Neueinstellungen: Eine empirische Untersuchung mit Personaldaten. Zeitschrift für Betriebswirtschaft, 79(5):633-662.

DOI: https://doi.org/10.1007/s11573-009-0243-z 


\section{Betriebsinterne Arbeitsmärkte, Hierarchien und Neueinstellungen: Eine empirische Untersuchung mit Personaldaten}
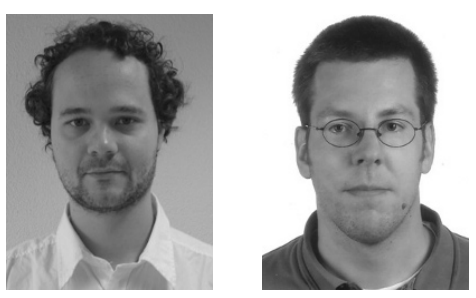

\section{Simon Janssen · Christian Pfeifer}

Zusammenfassung: In dieser Arbeit werden Personalstammdaten eines großen Unternehmens des verarbeitenden Gewerbes genutzt, in der die Belegschaft in Arbeiter, technische und kaufmännische Angestellte untergliedert ist. Im Sinne von Doeringer und Piore (1971) kann gezeigt werden, dass der interne Arbeitsmarkt für Arbeiter - insbesondere für Facharbeiter - wesentlich stärker vom externen Arbeitsmarkt isoliert ist, als dies bei Angestellten der Fall ist. Die Determinanten der Besetzung der Hierarchieebenen werden mit geordneten Probitmodellen untersucht. Die hierarchischen Positionen von Arbeitern werden stärker durch die Dauer der Betriebszugehörigkeit als Proxy für spezifisches Humankapital determiniert, während die Besetzung der Hierarchie bei Angestellten wesentlich durch formale Bildungsabschlüsse und allgemeine Erfahrung determiniert wird.

Schlüsselwörter: Effiziente Allokation · Interne Arbeitsmärkte · Personaldaten

JEL-Classification: $\mathrm{J} 41 \cdot \mathrm{M} 12 \cdot \mathrm{M} 51$

Eingegangen: 04.10 .2007

(C) Gabler-Verlag 2009

Für hilfreiche Anmerkungen danken wir Uschi Backes-Gellner, Knut Gerlach, Thomas Zwick, Jens Mohrenweiser, Donata Bessey, Margret Sterrenberg den Teilnehmern am Personalökonomischen Kolloquium 2007 in Tübingen und den Seminarteilnehmern an der Universität Zürich sowie dem Editor und zwei anonymen Gutachtern.

Dipl.-Ök S. Janssen $(\bowtie)$

Institut für Strategie und Unternehmensökonomik, Universität Zürich,

Plattenstr. 14, 8032 Zürich, Schweiz

E-Mail: simon.janssen@isu.unizh.ch

Dr. C. Pfeifer

Institut für Arbeitsökonomik, Leibniz Universität Hannover, Königsworther Platz 1,

30167 Hannover, Deutschland

E-Mail: pfeifer@aoek.uni-hannover.de 


\section{Motivation}

Wie Doeringer und Piore (1971) ausführen, ist die Kombination von betriebsspezifischem Humankapital und informellem „on the job“ Training der wesentliche Faktor für die Existenz eines internen geschlossenen Arbeitsmarktes. Informelles „on the job“ Training bezieht sich in diesem Sinne auf die Akkumulation von Humankapital, die direkt während der Arbeit stattfindet, zum Beispiel durch den direkten Umgang mit den Maschinen oder durch das Lernen von erfahrenen Kollegen während der normalen Arbeitstätigkeit. Darüber hinaus entsteht durch Investitionen in betriebsspezifisches Humankapital ein gegenseitiges Abhängigkeitsverhältnis zwischen der Firma und den Arbeitnehmern eines Betriebes (vgl. Becker, 1964). Spielen in einer Unternehmung spezifische Fähigkeiten eine besondere Rolle und werden diese hauptsächlich durch informelles „on the job“ Training erlernt, hängt der Wert des Humankapitals eines Beschäftigten vor allem von der Verweildauer innerhalb eines Betriebes ab. In diesem Sinne führt die Kombination dieser Faktoren zu Langzeitbeziehungen zwischen Arbeitgebern und Arbeitnehmern, die durch besondere administrative Regeln gekennzeichnet sind. Es entsteht ein interner Arbeitsmarkt innerhalb der Grenzen einer Firma.

Doeringer und Piore (1971) argumentieren, dass obige Faktoren zwar in allen Unternehmungen und für alle Beschäftigungsgruppen von Bedeutung sein können. Sie heben aber insbesondere hervor, dass spezifisches Humankapital, welches durch „on the job training" erworben wird, bei Arbeitern des verarbeitenden Gewerbes oder ähnlich strukturierten Wirtschaftsbereichen eine besondere Rolle spielt. Vor allem in der Stahl- und Chemieindustrie sollten laut den Autoren geschlossene interne Arbeitsmärkte innerhalb der Unternehmensgrenzen zu finden sein. Die Autoren nehmen hier eine eindeutige Unterscheidung zu Wirtschaftsbereichen wie dem Baugewerbe, dem Handwerk und dem Dienstleistungssektor sowie zu Angestellten vor. Zwar können laut den Autoren auch hier interne Arbeitsmärkte existieren, doch werden diese vielmehr durch geographische Gebiete, den gewerkschaftlichen Zuständigkeitsbereich oder die Branche abgegrenzt. Diese Bereiche werden nicht zwangsläufig alle typischen Merkmale geschlossener interner Arbeitsmärkte aufweisen. Insbesondere werden die internen Arbeitsmärkte weniger stark für externe Bewerber verschlossen sein.

Dieser Unterscheidung ist aber bisher in empirischen Untersuchungen kaum Beachtung geschenkt worden. So zeigt sich in vielen empirischen Arbeiten, die auf Doeringer und Piore (1971) Bezug nehmen, dass externe Bewerber auf nahezu allen Positionen der betriebsinternen Hierarchien eintreten. Eintrittspositionen im Sinne der Theorie von Doeringer und Piore (1971) können dagegen oftmals nicht bestätigt werden. Allerdings nutzt ein Großteil dieser Studien Daten von Banken, Dienstleistungsunternehmen oder Angestellten im gehobenen Management. Bei diesen Beschäftigten ist aber zu erwarten, dass die Kombination aus informellem „on the job“ Training und firmenspezifischem Humankapital eine geringere Rolle als im verarbeitenden Gewerbe spielt. Zwar werden auch Angestellte spezifisches Humankapital durch „on the job training“ erlernen, doch kommt hier allgemeinen Fähigkeiten, welche in Schule oder der akademischen Ausbildung erlernt wurden, eine wesentlich größere Bedeutung zu. Daher sind wesentliche Voraussetzungen für die Abschottung eines internen Arbeitsmarktes innerhalb solcher Unternehmung nicht in diesem Maße gegeben. 
In der vorliegenden Arbeit werden Daten verwendet, die es ermöglichen, die Beschäftigten eines Betriebes des verarbeitenden Gewerbes in Arbeiter, technische und kaufmännische Angestellte zu untergliedern. Es kann gezeigt werden, dass die Abschottung des internen Arbeitsmarktes der Arbeiter - und insbesondere der Facharbeiter - wesentlich stärker ausfällt als für die internen Arbeitsmärkte im Bereich der kaufmännischen und technischen Angestellten. Weiterhin zeigen die Ergebnisse, dass die Position innerhalb der betrieblichen Hierarchie bei Arbeitern vor allem durch die Dauer der Betriebszugehörigkeit beeinflusst wird, während die Betriebszugehörigkeit bei Angestellten keinen signifikanten Einfluss ausübt. Dieses Ergebnis kann als Indiz dafür gewertet werden, dass insbesondere bei den Arbeitern die Kombination aus firmenspezifischem Humankapital und informellem „on the job“ Training eine wesentliche Rolle spielt und zu einer Abschottung des internen Arbeitsmarktes führt.

Weiterhin zeigt sich, dass die hierarchische Position der Angestellten insbesondere durch formale Bildungsabschlüsse determiniert wird. Zwar ist die Wahrscheinlichkeit einen der oberen Ränge zu belegen für alle Beschäftigten am Anfang der Karriere recht gering. Für Akademiker steigt diese Wahrscheinlichkeit zum Ende des Arbeitslebens aber drastisch an. Die Chance der anderen Bildungsgruppen einen hohen Posten in der betrieblichen Hierarchie zu erreichen, bleibt dagegen über den gesamten Karriereverlauf auf einem vergleichsweise geringen Niveau. Da es sich bei der akademischen Ausbildung um eine formelle Ausbildung handelt, die weithin allgemeines Humankapital vermittelt, lässt sich schließen, dass diese Art von Humankapital im Bereich der Angestellten eine besondere Rolle spielt. Allgemeine Fähigkeiten aus formellen Ausbildungen sind nicht an ein bestimmtes Unternehmen gebunden und es lässt sich analog zu Doeringer und Piore (1971) erklären warum der Arbeitsmarkt der Angestellten weitaus weniger nach außen hin isoliert ist.

Der Beitrag gliedert sich wie folgt: Zunächst wird in Abschnitt 2 die Theorie betriebsinterner Arbeitsmärkte in Bezug auf Eintrittspositionen, spezifisches Humankapital, „on the job“"Training und hierarchische Strukturen erläutert. Abschnitt 3 gibt einen Überblick über den aktuellen Stand der empirischen Forschung. Abschnitt 4 beinhaltet Erläuterungen zum Personaldatensatz und deskriptive Auswertungen zur Besetzung der Hierarchieebenen. In Abschnitt 5 erfolgt eine multivariate Analyse der Besetzung von Hierarchieebenen. Den Abschluss bilden eine kurze Zusammenfassung und eine Diskussion, die vor allem der speziellen Situation des deutschen Arbeitsmarktes vor dem Hintergrund unserer Ergebnisse Rechnung trägt.

\section{Theorie betriebsinterner Arbeitsmärkte}

Doeringer und Piore (1971) folgend ist der externe Arbeitsmarkt der konventionelle, außerbetriebliche Arbeitsmarkt im Sinne der neoklassischen Theorie. Auf diesem Arbeitsmarkt steht die Arbeitsnachfrage einer großen Anzahl von Firmen einem sehr breiten Arbeitsangebot von vielen Arbeitssuchenden gegenüber. Somit sind die Möglichkeiten, Monopolmacht zu erlangen, sowohl für Firmen als auch für Arbeitnehmer eher gering (vgl. Wachter/Wright, 1990). Die Arbeitsbedingungen der Arbeitnehmer werden ständig neu verhandelt und die Lohnsetzung sowie die Allokation der Beschäftigten werden in 
erster Linie durch die klassischen Marktkräfte bestimmt. Aber bereits Kerr (1954) hob hervor, dass die Reichweite der kompetitiven Märkte aufgrund von Mobilitätskosten und arbeitsplatzspezifischen Eigenschaften eher gering ist und Langzeitbeziehungen zwischen Arbeitnehmern und Arbeitgebern eine besondere Rolle spielen.

Neben Faktoren wie Mobilitätskosten, Risikoaversion und Gewohnheit ist betriebsspezifisches Humankapital in Kombination mit „on the job“ Training für die Existenz interner Arbeitsmärkte in den Grenzen einer Unternehmung bestimmend. Da betriebsspezifische Kenntnisse und Fähigkeiten außerhalb des eigenen Betriebes wertlos sind, wird die Verhandlungsposition des Arbeitnehmers nach einer derartigen Investition deutlich geschwächt. Somit besteht die Gefahr, dass der jeweilige Arbeitgeber die Früchte einer solchen Investition erntet, ohne die Beschäftigten in angemessener Weise zu beteiligen oder diese gar im Nachhinein zu entlassen. Auf der anderen Seite kann eine vollständige Kostenübernahme durch den Arbeitgeber zu einer entgegengesetzten Situation führen, in der der Arbeitnehmer zu starke Verhandlungsmacht erlangt. Die aus der Literatur bekannte Lösung dieses Problems besteht in der Aufteilung der Kosten und Erträge zwischen Arbeitgeber und Arbeitnehmer (vgl. Becker, 1964). Hierdurch entstehen beiden Vertragspartnern versunkene Kosten, die einen Anreiz schaffen, eine Langzeitbeziehung zwischen Arbeitgeber und Arbeitnehmer aufrecht zu erhalten. Häufig können derartig spezifische Fähigkeiten nur „on the job“ vermittelt werden, beispielsweise während des Produktionsprozesses und durch die Weitergabe von implizitem Wissen durch erfahrene Mitarbeiter.

Um allerdings die komplexen wechselseitigen Beziehungen in einem dauerhaften Beschäftigungsverhältnis zu regeln, bedarf es administrativer Regeln und geordneter Abläufe. In diesem Sinne wies bereits Dunlop (1958) darauf hin, dass in jedem größeren Unternehmen, welches über einen längeren Zeitraum besteht, eine Reihe von administrativen Regeln und Abläufen existiert. Diese Regeln spielen vor allem in der Lohnsetzung, den Ausbildungsentscheidungen und der Allokation von Arbeitskräften eine besondere Rolle. Die Arbeitskräfte innerhalb des internen Arbeitsmarktes sind somit zumindest von den kurzfristigen Auswirkungen der ökonomischen Kräfte des Marktes isoliert. Wenn es unterschiedliche Beschäftigtengruppen innerhalb einer Unternehmung gibt, ist es überdies durchaus möglich, dass in derartigen Unternehmungen mehrere interne Arbeitsmärkte mit unterschiedlichen Strukturen entstehen. Dunlop (1988) hebt in diesem Sinne hervor, dass unterschiedliche Gruppen von Beschäftigten getrennt betrachtet werden müssen.

Trotz einer gewissen Abschottung von ökonomischen Einflüssen des externen Arbeitsmarktes ist der interne Arbeitsmarkt kein Konstrukt, welches eigenständig neben dem externen Arbeitsmarkt besteht (vgl. Wachter/Wright, 1990). Denn der interne Arbeitsmarkt interagiert über Ein- und Austrittspositionen (,Ports of Entry and Exit“") mit dem externen Arbeitsmarkt. Eintrittspositionen sind überwiegend Arbeitsplätze am unteren Ende der betrieblichen Hierarchie, welche den Beschäftigten ein geringeres Maß an Fähigkeiten abverlangen. Den Beschäftigten werden auf diesen Eintrittspositionen, vor allem auch durch „on the job“ Training, Fähigkeiten vermittelt, die für Tätigkeiten auf den weiterführenden Ebenen erforderlich sind. Können derartige Fähigkeiten außerhalb der Unternehmung nur schwer erlernt werden, führt dies dazu, dass die weiterführenden Positionen maßgeblich mit Beschäftigten aus den Eintrittspositionen besetzt werden. ${ }^{1}$ 
Doeringer und Piore (1971) argumentieren, dass die Arbeitskosten, insbesondere für Arbeiter des verarbeitenden Gewerbes, durch die Quantität in Zusammenhang mit der Qualität des Produktes determiniert werden. Qualitativ hochwertige Produkte in hoher Geschwindigkeit an meist auf bestimmte Produkte spezialisierten Maschinen zu erstellen, verlangt einen hohen Grad an spezifischen Fähigkeiten. Ferner stehen Produktionsabteilungen unter permanentem Druck, Kosten zu sparen. Dies führt dazu, dass sich die Produktionstechnologie ständig verändert und auf die speziellen Produkte zugeschnitten wird, um den Produktionsablauf effizienter zu gestalten. Letzteres geht wiederum mit spezifischem Wissen über die Technologie einher. Viele dieser technischen Veränderungen werden aber nicht detailliert dokumentiert und machen somit ein strukturiertes formales Erlernen dieses spezifischen Wissens schwer. Dies führt laut Doeringer und Piore (1971) dazu, dass insbesondere Produktionsarbeiter ihre Fähigkeiten „on the job“ erlernen müssen. Die Autoren bezeichnen Informalität in diesem Sinne als besonderes Kennzeichen des Trainings im verarbeitenden Gewerbe. Die Arbeiter werden daher früh am direkten Produktionsprozess beteiligt, um die Arbeitsabläufe zu erlernen. Außerdem ist die Akkumulation von Fähigkeiten oftmals entlang der Karriereleiter organisiert. Die Jobs am unteren Ende der Hierarchie vermitteln den Beschäftigten spezifische Fähigkeiten, welche diese auf den höheren Ebenen produktiv verwenden können. Liegen derartige Bedingungen vor, sollte der interne Arbeitsmarkt stark isoliert sein und über klar erkennbare Eintrittspositionen verfügen. Weiterhin ist zu erwarten, dass Humankapital vor allem durch den Verbleib in der Unternehmung akkumuliert wird und damit vor allem die Betriebszugehörigkeit bei der Allokation der Beschäftigten auf die einzelnen hierarchischen Ebenen eine besondere Rolle spielt.

Die Autoren betonen, dass auch bei Angestellten oder Beschäftigten des Managements spezifische Fähigkeiten von Bedeutung sind. Als Beispiele werden hier interaktive und kommunikative Fähigkeiten aufgeführt. Diese sind im Umgang mit Personen oder Teams innerhalb der Unternehmung von besonderer Bedeutung und können sich von Unternehmung zu Unternehmung wesentlich unterscheiden. Allerdings kommt bei dieser Beschäftigtengruppe auch generellen Fähigkeiten, die meist in formalen Ausbildungen vor Eintritt in das Berufsleben erworben werden, eine besondere Bedeutung zu. Überdies wird argumentiert, dass im Fall der Angestellten auch Fähigkeiten die nach Abschluss der Schulbildung erlernt werden oftmals allgemeinerer Natur sind. Vielfach werden diese Fähigkeiten sogar durch formelle Weiterbildungen, die auch außerhalb der Unternehmung stattfinden können, vermittelt. Derartige Fähigkeiten sind weniger stark an die einzelne Unternehmung gebunden und werden eher durch die Branche oder den Beruf begrenzt. Die Informalität der Ausbildung ist somit bei Arbeitern stärker ausgeprägt als bei Angestellten. Doeringer und Piore (1971) schließen daraus, dass „on the job“ Training bei Angestellten einen geringeren Stellenwert einnimmt und dass der interne Arbeitsmarkt der Angestellten weitaus stärker gegenüber dem externen Markt geöffnet ist. Denn das Wissen zwischen Unternehmungen einer Branche ist in starkem Maße zwischen einzelnen Betrieben transferierbar. Insbesondere wenn der eigene Beschäftigungspool einer Unternehmung nicht über genügend Mitarbeiter verfügt, die den schweren Aufgaben der Jobs auf den höheren Hierarchieebenen gewachsen sind, ist zu erwarten, dass externe Bewerber vermehrt auf höheren Ebenen einer betrieblichen Hierarchie eintreten. Doeringer und Piore (1971) führen aus, dass insbesondere für leitende Angestellte, Ingenieure und Wissenschaftler die 
formale berufliche Bildung und die allgemeine Berufserfahrung eine wichtigere Rolle spielen als die innerbetriebliche Karriere. Unter diesen Umständen ist zu erwarten, dass Angestellte mit höheren formalen Abschlüssen auf höheren hierarchischen Ebenen tätig sind, da sie über das nötige Humankapital verfügen, um die Aufgaben auf diesen Ebenen zu bewältigen. Außerdem wird der allgemeinen Berufserfahrung bei den Angestellten eine wesentlich größere Rolle zukommen als der Betriebszugehörigkeit, da ihr Wissen in stärkerem Maße zwischen den einzelnen Unternehmungen transferierbar ist.

Im Sinne der obigen Überlegungen lassen sich folgende Fragen stellen, die in der vorliegenden Arbeit anhand eines großen prozessproduzierten Personaldatensatzes untersucht werden. Ist der interne Arbeitsmarkt bei Arbeitern gegenüber externen Bewerbern geschlossener als bei Angestellten? Welche Faktoren beeinflussen die Besetzung der Hierarchie und wie lassen sich diese humankapitaltheoretisch interpretieren? In der letzten Frage soll hierbei insbesondere auf die Unterscheidung zwischen spezifischem und allgemeinem Humankapital eingegangen werden.

\section{Stand der empirischen Forschung}

Einen ausführlichen Überblick über die bestehende empirische Literatur zu internen Arbeitsmärkten liefert Lin (2005). Aus diesem Grund wird an dieser Stelle auf einen umfassenden Literaturüberblick verzichtet. Vielmehr werden die für diese Arbeit besonders relevanten Studien näher beleuchtet.

Die wohl bekanntesten und meistzitierten empirischen Arbeiten zu betriebsinternen Arbeitsmärkten stammen von Baker, Gibbs und Holmstrom (1994a; 1994b), die jährliche Personaldaten von Beschäftigten des Managements eines Dienstleistungsunternehmens über einen Zeitraum von 20 Jahren analysieren. Zwar finden einige wesentliche Merkmale des internen Arbeitsmarktes hier Bestätigung, wie beispielsweise Langzeitbeziehungen und stabile hierarchische Strukturen. Dennoch scheint die betrachtete Unternehmung nicht über einen vollständig geschlossenen Arbeitsmarkt zu verfügen, da externe Bewerber auf nahezu allen Ebenen eintreten. Diese Tatsache wird von den Autoren dahingehend interpretiert, dass keine Eintrittspositionen im Sinne von Doeringer und Piore (1971) existieren. Allerdings werden über 70 Prozent der Beschäftigten nicht aus dem externen Arbeitsmarkt rekrutiert, sondern in die jeweiligen Ebenen befördert. Die Produktivität der externen Bewerber unterscheidet sich nicht allzu stark von ihren internen Kollegen. Überdies konnte nicht gezeigt werden, dass spezifisches Humankapital eine besondere Rolle spielt.

Allerdings lassen sich auch zahlreiche andere Beispiele finden, in denen sich Ein- und Austritte über die gesamte Hierarchie der Unternehmen erstrecken. Lazear und Oyer (2004a; 2004b) untersuchen Angestellte mit schwedischen Daten. Die Autoren nutzen eine recht grobe Einteilung nach Berufsgruppen. Sie können aber zeigen, dass externe Eintritte in den von ihnen untersuchten Unternehmen auf nahezu allen Rängen stattfinden. Dennoch sind die Eintrittsraten auf den höheren Ebenen weitaus geringer und interne Beförderungen spielen gerade hier eine wesentliche Rolle. In Lazear und Oyer (2002) werden die zahlreichen Eintritte auf höheren Ebenen als Eintrittspositionen in gestaffelten Hierarchien interpretiert. Derartige Befunde könnten auch mit der Theorie getrennter Bereiche 
innerhalb eines betriebsinternen Arbeitsmarktes einhergehen (vgl. hierzu auch Lin, 2005). Ferner wird in Lazear und Oyer (2002) gezeigt, dass Einstellungen auf höheren Ebenen nur dann erfolgen, wenn dies mit Effizienzvorteilen verbunden ist. Derartige Effizienzvorteile dürften entstehen, wenn die externen Bewerber produktiver sind als ihre internen Konkurrenten. Chan (2006) und Acosta (2004) bestätigen in diesem Sinne, dass in den von ihnen untersuchten Firmen neu eingetretene Bewerber eine größere Chance haben, befördert zu werden. Chan (1996) führt dies darauf zurück, dass externe Bewerber von Firmen gehandikapt werden, um die Motivation der bereits im Unternehmen Tätigen zu sichern. Werden dennoch Externe eingestellt, müssen diese zwangsläufig besser sein. Evidenz für ein derartiges Handikap finden auch Agrawal, Knoeber und Tsoulouhas (2006). Mit Determinanten von Beförderungswahrscheinlichkeiten beschäftigt sich auch Lima (2000). In seiner Arbeit zeigt sich, dass Schulbildung einen positiven und die Betriebszugehörigkeit einen negativen Effekt auf die Beförderungswahrscheinlichkeit haben. Bewerber, die auf höheren Rängen in die Firma eintreten, verfügen ferner über mehr schulische Bildung als ihre intern beförderten Kollegen auf der gleichen Hierarchieebene. Köhler und Preisendörfer (1989) sowie Brüderl (1991) zeigen mit einem deutschen Datensatz, dass deutsche Männer eine höhere Beförderungswahrscheinlichkeit haben als Frauen.

Es ist zu beachten, dass ein Großteil der bestehenden Literatur selten Arbeiter und Angestellte derselben Unternehmung betrachtet. Überdies beinhalten viele Studien vor allem Unternehmungen aus dem Finanz- oder Dienstleistungssektor und beschränken sich dabei vornehmlich auf Angestellte oder Beschäftigte des Managements. Es lässt sich vermuten, dass in derartigen Unternehmungen und Bereichen die Abschottung des internen Arbeitsmarktes eine wesentlich geringere Rolle spielt. Die Studien von Lin (2005) sowie Dohmen, Kriechel und Pfann (2004) sind hier nennenswerte Ausnahmen. Beide Studien betrachten sowohl Angestellte als auch Arbeiter innerhalb einer Unternehmung. Überdies beschäftigen sich die Studien von Brüderl (1991) sowie Köhler und Preisendörfer (1989) separat mit Arbeitern.

Lin (2005) wertet Personaldaten eines taiwanesischen Autohändlers aus, dessen Belegschaft in kaufmännische Angestellte, Verkäufer und Werkstatttechniker untergliedert ist. Auch wenn er keine eindeutigen Eintrittspositionen findet, kann dennoch eine Konzentration der Eintritte am unteren Ende der Hierarchie identifiziert werden. So treten über die beobachteten Jahre zwischen 30 und 60 Prozent der Beschäftigten auf der untersten Ebene ein und Neueintritte werden mit zunehmender Ebene geringer. Vor allem Verkäufer steigen gegenüber Technikern auch vermehrt auf der höheren Ebene ein, was mit der besseren Verwertbarkeit von allgemeinem Humankapital begründet werden kann. Dohmen, Kriechel und Pfann (2004) analysieren Personaldaten von Arbeitern und Angestellten eines Unternehmens aus dem verarbeitenden Gewerbes. Sie können getrennte Eintrittspositionen auf den untersten Ebenen für Arbeiter und Angestellte ausmachen, obwohl auch hier auf höheren Ebenen einige Neueintritte stattfinden.

Auch für Deutschland liegt eine Studie von Oberst, Schank und Schnabel (2007) vor, in der einzelne Beschäftigtengruppen getrennt betrachtet werden. Die Beschäftigten werden in eine Rand- und Stammbelegschaft untergliedert. Es wird gezeigt, dass die Randbelegschaft - bestehend aus Leiharbeiten und Arbeitskräften mit befristeten Arbeitsverträgen - deutlich geringere Kosten verursacht als die Stammbelegschaft. Allerdings werden Eintrittspositionen und Karrierepfade hier nicht untersucht. Neben der Studie von 
Oberst, Schank und Schnabel (2007) liegen für Deutschland bisher nur wenige empirische Erkenntnisse aus der Analyse von prozessproduzierten Personaldaten vor. Als Ausnahmen wären noch Grund und Kräkel (2001) sowie Pfeifer (2008) zu nennen, die sich aber vor allem mit der Lohnsetzung auf internen Arbeitsmärkten beschäftigen.

\section{Datensatz und deskriptive Auswertungen}

\subsection{Der Datensatz}

Der Datensatz umfasst anonymisierte Personalstammdaten eines großen börsennotierten Unternehmens aus dem verarbeitenden Gewerbe. Das Unternehmen unterliegt einem Flächentarifvertrag, hat einen Betriebsrat und bietet innovative Produkte auf dem Weltmarkt an. Die Personaldaten wurden direkt aus dem Computersystem des Unternehmens exportiert und zur weiteren statistischen Auswertung aufbereitet. Sie enthalten monatliche Informationen über alle Beschäftigten im deutschen Stammwerk des Unternehmens von Januar 1999 bis Dezember 2005. Die folgenden Auswertungen erfolgen ohne Auszubildende, Praktikanten, Diplomanden sowie Beschäftigte in Altersteilzeit, da deren Stellung in der betrieblichen Hierarchie schwer auszumachen ist.

Ein wesentlicher Vorteil betrieblicher Personaldaten gegenüber anderen Individualdatenquellen (z. B. SOEP) besteht hinsichtlich der hohen Validität, da die Angaben exakt erfasst werden (vgl. Grund, 2002). In den meisten Datenquellen können zudem keine Arbeitnehmer desselben Betriebes und somit auch keine Hierarchien und innerbetrieblichen Karrieren identifiziert werden. Der zur Verfügung stehende Personaldatensatz enthält hingegen Informationen über die tariflichen Lohn- und Gehaltsgruppen der Beschäftigten, die Aufschluss über die Anforderungen der ausgeübten Tätigkeiten geben und als Proxy für die Hierarchieebene dienen. ${ }^{2}$ Tabelle 1 enthält eine Aufstellung der Hierarchieebenen laut Tarifvertrag. Es lassen sich drei getrennte Hierarchien mit mehreren Ebenen identifizieren. Die erste Hierarchie umfasst die Arbeiter, d. h. die Lohnempfänger. Die beiden anderen Hierarchien umfassen die technischen und kaufmännischen Angestellten, für die die Einteilung nach Ebenen identisch ist. Aufgrund geringer Fallzahlen in den untersten Lohn- und Gehaltsgruppen wurden diese zu einer Ebene zusammengefasst. Auch im außertariflichen Bereich (AT) ließen sich drei Gruppen unterscheiden, die jedoch im Einzelnen schwach besetzt sind. Die Abgrenzung der Hierarchieebenen über die Beschreibung der Lohn- und Gehaltsgruppen umfasst somit eine Abgrenzung zwischen Hilfsarbeitern (Arbeiter in Ebenen 1 bis 3) und Facharbeitern (Arbeiter in Ebenen 4 bis 7) sowie Angestellten im niedrigen (Angestellte in Ebenen 1 bis 2), mittleren (Angestellte in Ebenen 3 bis 5) und oberen (Angestellte in Ebene 6) Bereich.

\subsection{Deskriptive Resultate}

Tabelle 2 enthält zunächst einige deskriptive Informationen zu Arbeitern und ihren sieben Hierarchieebenen über den gesamten Beobachtungszeitraum. Insgesamt liegen 52002 monatliche Beobachtungen vor. Im Durchschnitt sind 627 Arbeiter im Unternehmen tätig, von denen die meisten Facharbeiter sind (ab Ebene 4). Für sie ergibt sich eine pyrami- 
Tab. 1: Einteilung in Hierarchieebenen gemäß Tarifvertrag

\begin{tabular}{|c|c|c|}
\hline Ebene & Arbeiter & technische und kaufmännische Angestellte \\
\hline 1 & $\begin{array}{l}\text { einfache Arbeiten (Unterweisung, Anler- } \\
\text { nen), Lohngruppen } 1 \text { bis } 4\end{array}$ & $\begin{array}{l}\text { einfachere Tätigkeiten, Gehaltsgruppen } 1 \\
\text { bis } 3\end{array}$ \\
\hline 2 & $\begin{array}{l}\text { mittelschwere Arbeiten (Sonderausbil- } \\
\text { dung), Lohngruppe } 5\end{array}$ & $\begin{array}{l}\text { Tätigkeiten mit erhöhten Anforderungen } \\
\text { (ordnungsgemäße Berufsausbildung), Ge- } \\
\text { haltsgruppe } 4\end{array}$ \\
\hline 3 & $\begin{array}{l}\text { mittelschwere Arbeiten (zweijährige Aus- } \\
\text { bildung), Lohngruppe } 6\end{array}$ & $\begin{array}{l}\text { schwierige Tätigkeiten (Fachhochschulab- } \\
\text { schluss), Gehaltsgruppe } 5\end{array}$ \\
\hline 4 & $\begin{array}{l}\text { Facharbeiten (ordnungsgemäße Berufsaus- } \\
\text { bildung), Lohngruppe } 7\end{array}$ & $\begin{array}{l}\text { schwierige Tätigkeiten und Entscheidungen } \\
\text { im eigenen Aufgabenbereich (Hochschulab- } \\
\text { schluss), Gehaltsgruppe } 6\end{array}$ \\
\hline 5 & $\begin{array}{l}\text { schwierige Facharbeiten (ordnungsgemäße } \\
\text { Berufsausbildung), Lohngruppe } 8\end{array}$ & $\begin{array}{l}\text { sehr schwierige Tätigkeiten und Entschei- } \\
\text { dungen von erheblicher Bedeutung (Hoch- } \\
\text { schulabschluss), Gehaltsgruppe } 7\end{array}$ \\
\hline 6 & $\begin{array}{l}\text { besonders schwierige oder hochwertige } \\
\text { Facharbeiten (ordnungsgemäße Berufsaus- } \\
\text { bildung), Lohngruppe } 9\end{array}$ & $\begin{array}{l}\text { außertariflich Beschäftigte, AT-1 bis AT-3 } \\
\text { ohne Vorstand }\end{array}$ \\
\hline 7 & $\begin{array}{l}\text { hochwertigste Facharbeiten (ordnungsge- } \\
\text { mäße Berufsausbildung), Lohngruppe } 10\end{array}$ & \\
\hline
\end{tabular}

denförmige Struktur, da der Anteil an den Beschäftigten ab Hierarchieebene 5 abnimmt. Wie auch in den Untersuchungen von Dohmen, Kriechel und Pfann (2004), Baker, Gibbs und Holmstrom (1994a; 1994b) oder auch Lima (2000) lassen sich auch bei uns keine eindeutigen Eintrittspositionen fest machen. Dennoch fällt auf, dass auf der ersten und der vierten Ebene überproportional viele Einstellungen stattfinden. So wurden fast 20 Prozent der Arbeiter auf Ebene 1 und 65 Prozent auf Ebene 4 eingestellt. Diese Tatsache kann als Indiz für einen zweigeteilten internen Arbeitsmarkt innerhalb der betrieblichen Hierarchie der Arbeiter gewertet werden. Die ersten drei Ebenen wären in diesem Sinne vor allem den schlechter ausgebildeten Arbeitern vorbehalten. Es zeigt sich ferner, dass die ersten drei Ebenen nicht der Struktur einer Pyramide folgen. Die Ebenen 4 bis 7 sind hingegen auch im Sinne der Definition des Tarifvertrages vor allem mit ausgebildeten Facharbeitern besetzt. Es fällt überdies auf, dass in Ebene 6 und 7 keine Eintritte mehr statt finden. Dies steht im Gegensatz zu vielen anderen empirischen Studien (vgl. bspw. die Übersicht von Lin, 2005). Es allerdings anzumerken, dass die hierarchischen Strukturen zwischen den einzelnen Unternehmen eher schwer zu vergleichen sind. Dennoch kann geschlossen werden, dass der interne Arbeitsmarkt der Facharbeiter in der von uns betrachteten Unternehmung vergleichsweise stark nach außen abgeschottet ist.

Betrachtet man vor diesem Hintergrund zwei getrennte interne Arbeitsmärkte als eigenständig, würden in der Hierarchie der geringqualifizierten Arbeiter (Ebenen 1 bis 3) 64 Prozent aller neuen Beschäftigten auf Ebene 1 eintreten, 4 Prozent auf Ebene 2 und 32 Prozent auf Ebene 3. In der Hierarchie der Facharbeiter (Ebenen 4 bis 7) würden hingegen 91 Prozent der Neuzugänge auf Ebene 4 und nur 8 Prozent der Neuzugänge auf Ebene 5 eintreten. In den Ebenen 6 und 7 fanden während des Beobachtungszeitraumes keine Eintritte statt. In diesem Sinne scheint der interne Arbeitsmarkt für geringqualifizierte Arbeiter weniger stark vom externen Markt abgeschottet zu sein, als der für die Facharbeiter. 
Insbesondere für den ersteren Markt könnte man die Werte als ähnlich zu den Befunden von Baker, Gibbs und Holmstrom (1994a) bezeichnen, welche als nicht vorhandene Eintrittspositionen ausgelegt werden. Dies erscheint auch plausibel, wenn es sich bei den Geringqualifizierten um eine flexible Randbelegschaft handelt. Der interne Arbeitsmarkt für Facharbeiter ist jedoch vergleichsweise stark vom externen Arbeitsmarkt isoliert.

Während auf den Ebenen 1 bis 3 lediglich die Hälfte der Arbeiter eine Berufsausbildung absolviert hat, sind dies auf den höheren Ebenen deutlich mehr. Auf Ebene 4 steigt der Anteil der ausgebildeten Arbeiter um das 1,3-fache der dritten Ebene an. Die Unterschiede beim Alter fallen eher gering aus. Dagegen sind die Unterschiede bei der Länge der Betriebszugehörigkeit beträchtlich. Insbesondere auf Ebene 1 und Ebene 4 ist die durchschnittliche Länge der Betriebszugehörigkeit deutlich geringer als auf den nach gelagerten Ebenen. Auch diese Ergebnisse sprechen dafür, dass der interne Arbeitsmarkt der Arbeiter als zweigeteilt zu betrachten ist.

Abgesehen von den Neueinstellungen auf Ebene 1 verfügen alle neuen Arbeiter über eine Berufsausbildung. Dieser Befund kann teilweise einem qualifikationsverzerrenden technischen Fortschritt (z. B. Bedienung von komplexen Maschinen) zugerechnet werden, der die Einstellung höher qualifizierter Arbeiter erfordert. Zudem entspricht das Ergebnis der Definition der Hierarchieebenen im Tarifvertrag, der bereits ab Ebene 2 eine Sonderausbildung vorsieht. Jedoch können Arbeiter mit fehlenden formalen Qualifikationen im Laufe ihrer Betriebszugehörigkeit dazulernen und auch in der Hierarchie aufsteigen. Ähnliche Ergebnisse finden auch Baker, Gibbs und Holmstrom (1994a). Auch hier zeigt sich, dass die Schulbildung der Neuzugänge auf den einzelnen Ebenen ein wenig höher ist, als die der in die einzelnen Levels beförderten Personen. Lima (2000) zeigt ein vergleichbares, wenn auch weniger eindeutiges Muster. Insbesondere auf den Ebenen 4 und 5 in der von uns betrachteten Unternehmung sind die Bildungsunterschiede zwischen Neuzugängen und Personen, die in diese Hierarchieebenen befördert werden, auch im Vergleich zu vorhergehenden empirischen Studien recht deutlich. Dieses könnte durch vergleichsweise rigide Einstellungskriterien die sich auf die institutionellen Regeln des Tarifvertrags beziehen zurückgeführt werden.

Die letzten Spalten in Tab. 2 enthalten Informationen zu Beförderungen. Im gesamten Beobachtungszeitraum sind 158 Arbeiter befördert worden, d. h. es wurden fast doppelt so viele Arbeiter befördert wie neu eingestellt. Hieraus lässt sich schließen, dass die Mehrzahl der freien Stellen mit Insidern besetzt wurde. Eine separate Auswertung nach Ebenen zeigt, dass auf den Ebenen 2 und 3 mehr Arbeiter aus einer unteren Ebene befördert als neu eingestellt wurden. Auf der Einstiegsebene für Facharbeiter (Ebene 4) erfolgten mehr Neueinstellungen als Beförderungen, während auf höheren Ebenen fast alle Arbeiter aus unteren Ebenen befördert wurden. Insgesamt kann dieser Befund als ein recht starkes Indiz für die Existenz von Eintrittspositionen und betrieblichen Karrieren gewertet werden. Eine derartig deutliche Besetzung der Hierarchieebenen mit Insidern lässt sich bspw. weder bei Lima (2000) noch bei Baker, Gibbs und Holmstrom (1994a; 1994b) finden.

Die deskriptiven Auswertungen für technische und kaufmännische Angestellte sind in Tab. 3 zusammengefasst. Im Durchschnitt sind ungefähr 58 Prozent der Angestellten (530 Beschäftigte) im technischen Bereich und 42 Prozent im kaufmännischen Bereich (388 Beschäftigte) tätig. Da im Gegensatz zu den Arbeitern auf allen Ebenen Neueinstel- 


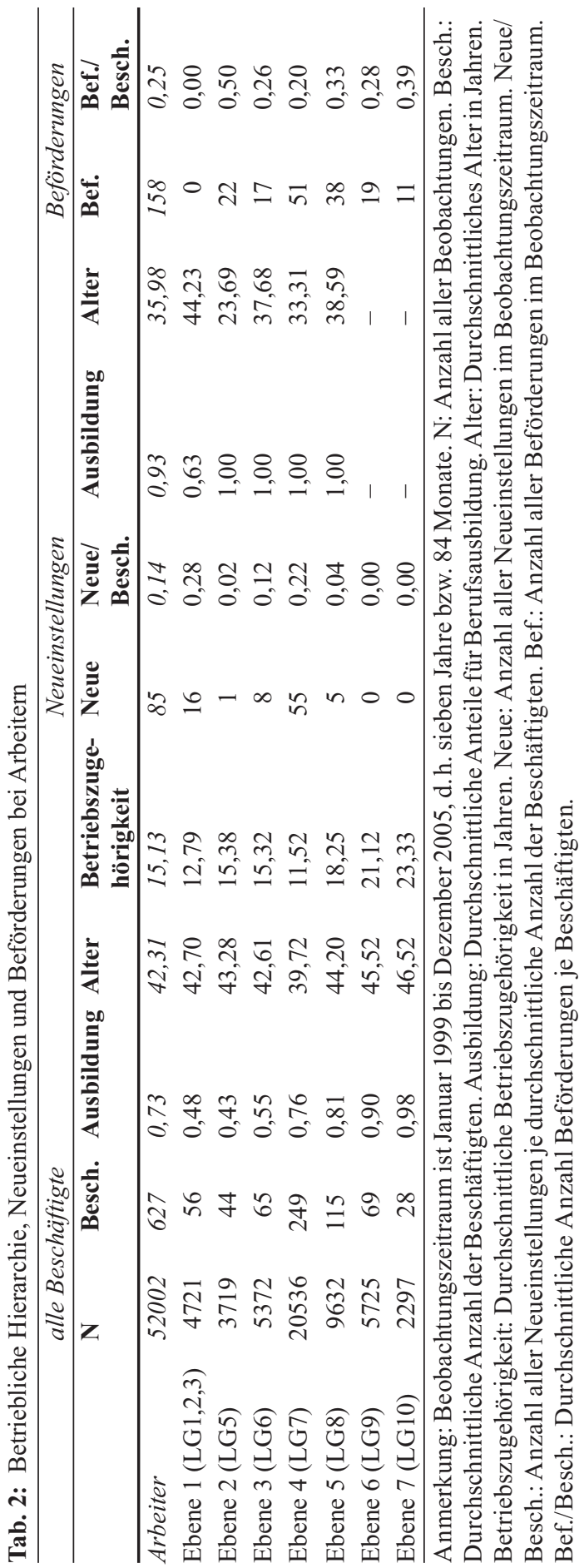




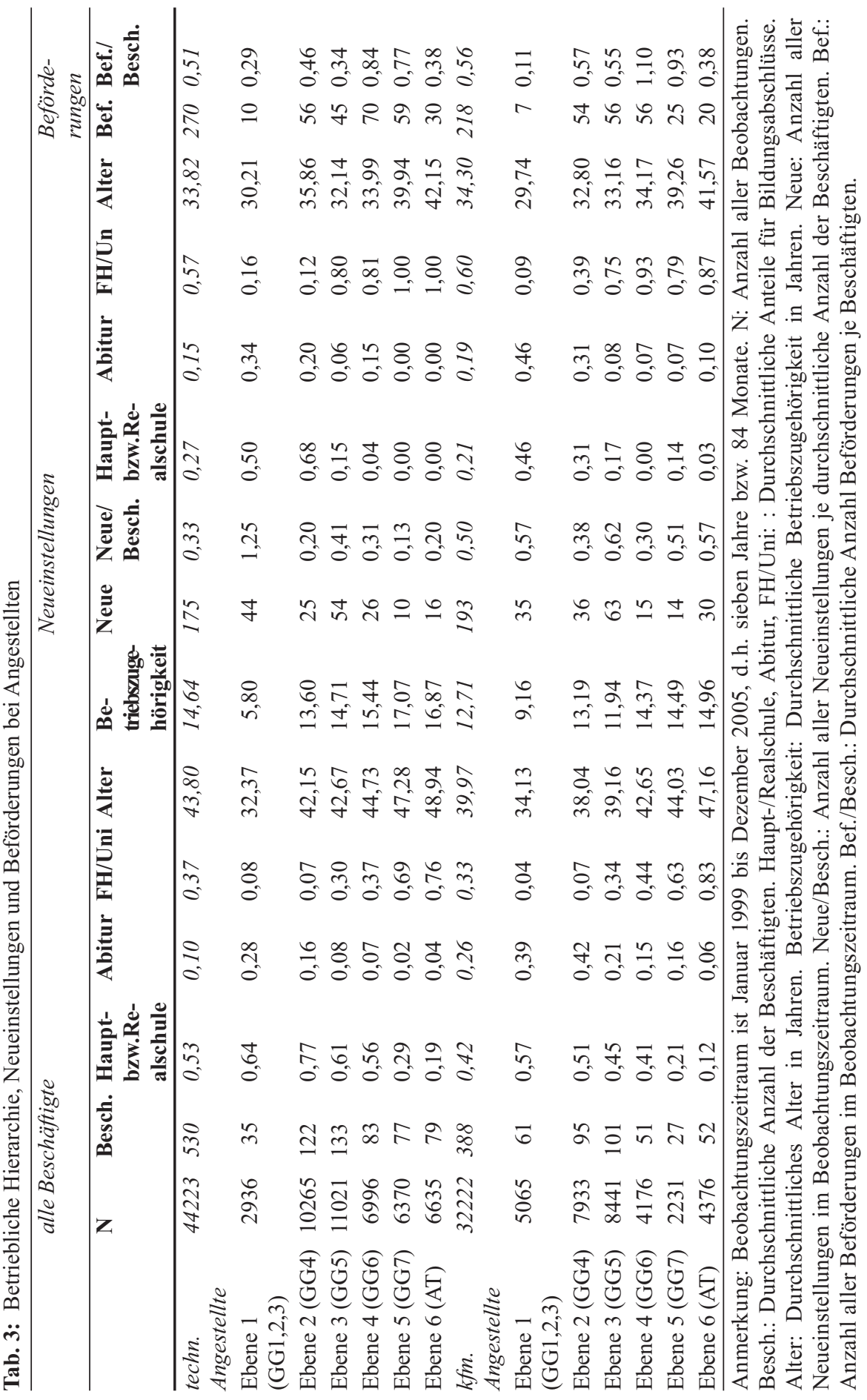


lungen stattfinden und auch das Verhältnis von Neueinstellungen zur durchschnittlichen Anzahl der Beschäftigten deutlich höher ist, ist die Abschottung vom externen Arbeitsmarkt somit bei Angestellten wesentlich schwächer als bei Arbeitern. Aber auch bei Angestellten finden auf zwei Ebenen überproportional viele Neueinstellungen statt. Diese abgeschwächten Eintrittspositionen sind zum einen die Ebene 1 für geringer qualifizierte Angestellte im niedrigen Angestelltenbereich und zum anderen die Ebene 3 für höher qualifizierte Angestellte im mittleren Angestelltenbereich. Für technische und kaufmännische Angestellte liegen die Ergebnisse vergleichsweise näher an den Ergebnissen von Baker, Gibbs und Holmstrom (1994a). Auch hier steigen auf allen Ebenen neue Personen in das Unternehmen ein, obwohl interne Karrieren dennoch eine Rolle zu spielen. Jedoch fällt die Abschottung vom externen Markt insgesamt schwächer aus.

Die Anteile der Beschäftigten nach Bildungsabschlüssen (Haupt- oder Realschule, Abitur, Fachhochschul- oder Universitätsabschluss) zeigen, dass Angestellte auf höheren Ebenen im Durchschnitt höhere formale Bildungsabschlüsse besitzen. Auch hier scheint es eine Schwelle zu geben, da der Anteil der Akademiker von der zweiten auf die dritte Ebene um das ca. 4,3-fache ansteigt. Anzumerken ist ferner, dass in Ebene 2 bei den Neueinstellungen der kaufmännischen Angestellten 39 Prozent und bei den technischen Angestellten 12 Prozent einen akademischen Abschluss besitzen und selbst auf Ebene 1 noch Akademiker eingestellt werden. Dies zeigt, dass ein akademisches Bildungssignal zwar die Wahrscheinlichkeit stark erhöht in eine der höheren Ebenen zu gelangen, es bringt aber keinen garantierten Anspruch auf eine bestimmte Position mit sich. Im Unterschied zu Arbeitern sind Angestellte auf höheren Ebenen deutlich älter als auf niedrigeren Ebenen. Auch die durchschnittliche Länge der Betriebszugehörigkeit nimmt tendenziell mit der Ebene zu. Jedoch ist die Differenz zwischen den Ebenen deutlich geringer als bei Arbeitern. Ebenso wie bei Arbeitern sind die neu eingestellten Angestellten im Durchschnitt höher qualifiziert und jünger als die Gesamtheit der Angestellten. Die Befunde deuten darauf hin, dass neu eingestellte Arbeitnehmer fehlendes spezifisches Humankapital teilweise durch mehr allgemeines Humankapital kompensieren können. Auch bei den Angestellten fallen die Bildungsunterschiede stärker als bei Baker, Gibbs und Holmstrom (1994a) aus. So ist bereits auf den unteren Ebenen der Anteil der Neueinsteiger mit einem Universitätsabschluss doppelt so groß wie der Anteil der intern Beförderten, die über einen Universitätsabschluss verfügen. In Baker, Gibbs und Holmstrom (1994a) unterscheiden sich die intern beförderten und extern rekrutierten Arbeiter nur um circa ein halbes Schuljahr. Wenn das deutsche Schulsystem auch nicht direkt mit dem amerikanischen vergleichbar ist, deutet doch einiges darauf hin, dass die Bildungsunterschiede zwischen Internen und Externen in der von uns betrachteten Unternehmung deutlich größer sind. In Lima (2000) sind die Bildungsunterschiede zwischen intern beförderten Personen und extern Rekrutierten Personen sehr variabel und unterscheiden sich von Level zu Level recht stark. Ein so eindeutiges Bild wie im vorliegenden Fall ergibt sich nicht. Abschließend kann daher gesagt werden, dass in der von uns betrachteten Unternehmung sowohl bei Arbeitern als auch bei Angestellten die Bildungsunterschiede zwischen Inund Outsideren vergleichsweise groß ausfallen. Es lässt sich vermuten, dass dieses Ergebnis auf die institutionellen Rahmenbedingungen in Deutschland zurück zu führen ist. In diesem Sinne wird die Einstellungspolitik der Unternehmen stark von den Ausführungen des Tarifvertrages beeinflusst. 
Wie bei den Arbeitern gibt es sowohl bei den technischen als auch bei den kaufmännischen Angestellten mehr Beförderungen als Neueinstellungen. Zwar ist das Verhältnis von Beförderungen zu Neueinstellungen nicht ganz so groß wie bei den Arbeitern, aber das Verhältnis zu der durchschnittlichen Anzahl der Beschäftigten ist hier doppelt so groß, was auf eine höhere Dynamik im Arbeitsmarkt der Angestellten hinweist. Bei separaten Auswertungen für die einzelnen Ebenen zeigt sich ein ähnliches Muster wie zuvor. Auf den Einstiegsebenen (Ebenen 1 und 3) finden mehr Neueinstellungen als Beförderungen und auf den jeweils nachgelagerten Ebenen tendenziell mehr Beförderungen als Neueinstellungen statt.

Interessanterweise scheinen in dem von uns betrachteten Unternehmen relativ deutliche Eintrittspositionen für Arbeiter - insbesondere für Facharbeiter - zu existieren. Die Abschottung der Angestellten vom externen Arbeitsmarkt ist dagegen deutlich schwächer, aber dennoch in bestimmten Grenzen zu beobachten. Die Resultate kommen den Ausführungen von Doeringer und Piore (1971) relativ nahe. Die deskriptiven Auswertungen zeigen weiterhin für alle drei Gruppen, dass innerbetrieblichen Karrieren in der Hierarchie große Bedeutung zukommt. Neu eingestellte Arbeitnehmer sind im Durchschnitt jünger, die durchschnittliche Länge der Betriebszugehörigkeit ist recht hoch und nimmt mit der Hierarchieebene zu. Es gibt mehr Beförderungen als Neueinstellungen. Zudem stellt das Unternehmen Arbeitnehmer mit höheren formalen Bildungsabschlüssen ein als bereits beschäftigte Arbeitnehmer auf der gleichen Ebene haben. Mögliche ökonomische Gründe für diesen Befund könnten zum einen darin liegen, dass das Untenehmen formale Bildungsabschlüsse als Signal nutzt, um neu eingestellte Arbeitnehmer in die Hierarchie einzuordnen. Nach Chan (1996) kann zum anderen eine alternative Interpretation im Zusammenhang mit der Anreizgestaltung im Rahmen von Beförderungsturnieren erfolgen. Um einen Effizienzverlust in der Anreizsetzung auszugleichen, muss ein auf einer höheren Ebene eingestellter - und somit einem Insider vorgezogener - Arbeitnehmer produktiver sein als der nicht beförderte Insider. Ein höherer Bildungsabschluss kann dementsprechend als Proxy für die Produktivität gewertet werden. Diese Überlegung wird eher dann zutreffen, wenn Fähigkeiten von allgemeiner Natur sind.

In Tab. 4 stellen wir die hierarchische Struktur zu Beginn unseres Beobachtungszeitraumes (Januar 1999) und zum Ende (Dezember 2005) gegenüber. Die Anzahl der Beschäftigten in den beiden Monaten unterscheiden sich nur geringfügig, sind aber deutlicher geringer als die durchschnittliche Anzahl der Beschäftigten (vgl. Tab. 2 und 3), da das Unternehmen zwischenzeitlich eine Expansionsphase und dann eine Konsolidierungsphase durchlaufen hat. Der Anteil der Arbeiter an allen Beschäftigten ist etwas zurückgegangen, während der Anteil der kaufmännischen Angestellten leicht gestiegen ist. Die technischen Angestellten unterlagen kaum Veränderungen. Innerhalb der Hierarchie der Arbeiter gab es eine Verschiebung von geringqualifizierten Arbeitern zu Facharbeitern (insbesondere auf Ebene 4). Auch bei kaufmännischen Angestellten ist der Anteil der Ebenen 1 und 2 für geringer Qualifizierte gesunken und der Anteil der Ebenen des mittleren und höheren Angestelltenbereichs gestiegen. Es zeigt sich also auch hier eine Tendenz hin zu höher qualifizierten Arbeitnehmern, wodurch die Überlegungen zum qualifikationsverzerrenden Fortschritt unterstützt werden. Vergleicht man unsere Auswertungen bspw. mit denen von Dohmen, Kriechel und Pfann 
Tab. 4: Vergleich der Hierarchie Januar 1999 und Dezember 2005

\begin{tabular}{lrrrrrr}
\hline & \multicolumn{5}{c}{ Januar 1999 } & \multicolumn{5}{c}{ Dezember 2005 } \\
\hline & \multicolumn{1}{c}{ N } & Anteil in \% & Kumuliert & \multicolumn{1}{c}{ N } & Anteil in \% & Kumuliert \\
\hline Arbeiter & 581 & 100,00 & & 563 & 100,00 & \\
Ebene 1 & 60 & 10,33 & 10,33 & 51 & 9,06 & 9,06 \\
(LG1,2,3,4) & & & & & & \\
Ebene 2 (LG5) & 44 & 7,57 & 17,90 & 44 & 7,82 & 16,87 \\
Ebene 3 (LG6) & 82 & 14,11 & 32,01 & 50 & 8,88 & 25,75 \\
Ebene 4 (LG7) & 182 & 31,33 & 63,34 & 245 & 43,52 & 69,27 \\
Ebene 5 (LG8) & 112 & 19,28 & 82,62 & 101 & 17,94 & 87,21 \\
Ebene 6 (LG9) & 77 & 13,25 & 95,87 & 50 & 8,88 & 96,09 \\
Ebene 7 (LG10) & 24 & 4,13 & 100,00 & 22 & 3,91 & 100,00 \\
techn. Angestellte & 484 & 100,00 & & 499 & 100,00 & \\
Ebene 1 (GG1,2,3) & 20 & 4,13 & 4,13 & 36 & 7,21 & 7,21 \\
Ebene 2 (GG4) & 118 & 24,38 & 28,51 & 118 & 23,65 & 30,86 \\
Ebene 3 (GG5) & 130 & 26,86 & 55,37 & 106 & 21,24 & 52,10 \\
Ebene 4 (GG6) & 80 & 16,53 & 71,90 & 86 & 17,23 & 69,34 \\
Ebene 5 (GG7) & 56 & 11,57 & 83,47 & 72 & 14,43 & 83,77 \\
Ebene 6 (AT) & 80 & 16,53 & 100,00 & 81 & 16,23 & 100,00 \\
kfm. Angestellte & 329 & 100,00 & & 374 & 100,00 & \\
Ebene 1 (GG1,2,3) & 54 & 16,41 & 16,41 & 51 & 13,64 & 13,64 \\
Ebene 2 (GG4) & 92 & 27,96 & 44,38 & 82 & 21,93 & 35,56 \\
Ebene 3 (GG5) & 82 & 24,92 & 69,30 & 107 & 28,61 & 64,17 \\
Ebene 4 (GG6) & 39 & 11,85 & 81,16 & 50 & 13,37 & 77,54 \\
Ebene 5 (GG7) & 18 & 5,47 & 86,63 & 31 & 8,29 & 85,83 \\
Ebene 6 (AT) & 44 & 13,37 & 100,00 & 53 & 14,17 & 100,00 \\
\hline
\end{tabular}

(2004), deutet einiges darauf hin, dass die Veränderungen der hierarchischen Struktur in der von uns betrachteten Unternehmung ein wenig stärker ausfallen. Obwohl die Auswertungen von Dohmen, Kriechel und Pfann (2004) unseren Auswertungen auf Grund der Datenbasis recht nah liegen, muss jedoch erwähnt werden, dass die Hierarchieebenen der beiden Studien nicht direkt miteinander verglichen werden können. Es lässt sich jedoch sagen, dass die hierarchische Struktur in Dohmen, Kriechel und Pfann (2004) trotz starker wirtschaftlicher Schwankungen erstaunlich stabil bleibt, wohingegen sich in unseren Daten kleinere Verschiebungen feststellen lassen. Dennoch kann nicht behauptet werden, dass unsere Ergebnisse in der Tendenz maßgeblich von ihren Auswertungen oder den Auswertungen anderer Studien (z. B. Lima (2000) oder Baker, Gibbs und Holmstrom (1994a; 1994b)) abweichen. Insgesamt wird das in der Literatur oftmals anzutreffende Ergebnis stabiler hierarchischer Strukturen auch von unseren Betrachtungen gestützt. 


\section{Multivariate Analyse}

\subsection{Methodik}

Nachdem wir im vorherigen Abschnitt bereits einige deskriptive Informationen zu den einzelnen Hierarchien und Neueintritten in dem von uns betrachteten Unternehmen geliefert haben, erfolgt an dieser Stelle eine multivariate Analyse der Besetzung von Hierarchieebenen. Zum einen soll untersucht werden, ob spezifisches Humankapital in den einzelnen Bereichen eine Rolle spielt. Zum anderen können die Karriereverläufe der einzelnen Bildungsgruppen betrachtet werden. Zu diesem Zweck werden geordnete Probitmodelle mit der Hierarchieebene als abhängiger Variable geschätzt. Alle Schätzungen werden als Querschnittsschätzungen mit robusten Standardfehlern durchgeführt. Den geordneten Probitmodellen liegt folgendes latentes Modell zugrunde:

$$
y^{*}=\beta_{1} A+\beta_{2} A^{2}+\beta_{3} T+\beta_{4} T^{2}+\beta_{5} G+\beta_{6} E+\sum_{k} \beta_{k} B_{k}+\varepsilon
$$

Wobei

$$
\begin{aligned}
& y=1 \text { wenn }-\infty<y^{*} \leq \mu_{1} \\
& y=2 \text { wenn } \mu_{1}<y^{*} \leq \mu_{2} \\
& y=j \text { wenn } \mu_{j-1}<y^{*} \leq \infty
\end{aligned}
$$

gilt $^{3}$

$\varepsilon$ sei ein standardnormalverteilter Störterm, $y^{*}$ die zugrunde liegende nicht beobachtbare latente Variable. Die Variable $y$ bezieht sich auf die jeweilige hierarchische Ebene von 1 bis $j$. Als erklärende Variablen dienen verschiedene individuelle Informationen über die Beschäftigten (vgl. Tab. A.1. im Anhang). Mit $B_{k}$ werden binäre Variablen für den höchsten Bildungsabschluss als Indikator für allgemeines schulisches Humankapital und als Indikator für die Fähigkeit am Arbeitsplatz zu lernen aufgenommen (vgl. Gibbons/Waldman, 1999; 2006). Da Arbeiter fast ausschließlich Haupt- und Realschulabschlüsse besitzen, kann hier nur zwischen Arbeitern mit und ohne Berufsausbildung unterschieden werden. Bei Angestellten existiert dagegen eine größere Variation der Bildungsabschlüsse. Hier besteht jedoch das Problem, dass für Akademiker die Information zur Berufsausbildung nicht verfügbar ist. Daher trennen wir bei Angestellten lediglich zwischen Haupt- bzw. Realschulabschluss, Abitur und Fachhochschul- bzw. Universitätsabschluss. Ferner beziehen wir mit $A$ das in Jahren gemessene Alter und die Dauer der Betriebszugehörigkeit $T$ der Beschäftigten mit ein. Aufgrund von möglichen Nichtlinearitäten nehmen wir auch deren quadrierte Terme $A^{2}$ und $T^{2}$ auf. In zusätzlichen Schätzungen hat sich gezeigt, dass Terme höherer Ordnung insignifikant waren. Das Alter ist ein Indikator für erworbenes allgemeines Humankapital durch Berufs- und Lebenserfahrung. Die Dauer der Betriebszugehörigkeit ist ein Indikator für erworbenes betriebsspezifisches Humankapital, vor allem dann, wenn es hauptsächlich im Zeitablauf „on the job“ erworben wird. Nach obigen Ausführungen ist zu erwarten, dass insbesondere bei den Arbeitern die Betriebszugehörigkeit eine besondere Rolle spielt, wohingegen diese 
Variable bei den Angestellten von nachrangiger Bedeutung sein sollte. Weiterhin wird für mögliche geschlechtsspezifische Effekte kontrolliert, indem eine binäre Variable $G$ aufgenommen wird, die den Wert Eins annimmt, falls es sich um eine weibliche Beschäftigte handelt. Abschließend wird eine binäre Variable $E$ berücksichtigt, die angibt, ob der Beschäftigte im laufenden Monat in den Betrieb eingestiegen ist. Durch $\mu_{1}$ bis $\mu_{j}$ werden die jeweiligen Schwellenwerte beschrieben.

\subsection{Ergebnisse der multivariaten Analyse}

Tabelle 5 informiert über die Ergebnisse der geordneten Probit Schätzungen zur Besetzung der Hierarchieebenen bei Arbeitern. Wir haben fünf Spezifikationen geschätzt. Es ist zu erwähnen, dass sich die marginalen Effekte der jeweiligen Variablen zwischen den einzelnen Hierarchieebenen unterscheiden und von der Richtung des Koeffizienten nicht direkt auf das Vorzeichen der marginalen Effekte in den einzelnen Ebenen geschlossen werden kann. Dennoch kann beispielsweise ein positiver Koeffizient als eine Art übergreifender positiver Effekt interpretiert werden. Ein Anstieg einer Variablen mit einem positiven Koeffizienten würde in diesem Sinne bedeuten, dass die ursprüngliche Verteilung von einer derartig veränderten Verteilung statistisch dominiert wird. Ein positiver Koeffizient kann demnach als eine Tendenz in Richtung der höchsten Hierarchieebene interpretiert werden.

Die erste Spezifikation zeigt, dass Frauen und Arbeitnehmer ohne abgeschlossene Berufsausbildung auf geringeren Ebenen tätig sind $(\mathrm{p}<0,01)$. Das Alter hat im Gegensatz zur Dauer der Betriebszugehörigkeit keinen signifikanten Einfluss. Der positive Effekt der Betriebszugehörigkeit deutet darauf hin, dass betriebliche Karrieren mit Aufstiegen in der Hierarchie existieren. Zudem kann dies als Indiz für die Bedeutung von betriebsspezifischem Humankapital und „on the job“ Training gedeutet werden. In der zweiten Spezifikation betrachten wir den Einfluss von Betriebszugehörigkeit isoliert und lassen das Alter aus der Schätzung. Die Ergebnisse bleiben hiervon jedoch unbeeinflusst. Die dritte Spezifikation nimmt nun das Eintrittsalter der Arbeiter mit auf, welches einen signifikant negativen Einfluss hat. Dieser Befund zeigt, dass vor Eintritt in das Unternehmen erworbene allgemeine Qualifikationen nicht von großem Wert in der Firma sind. Ferner haben ältere Personen eine kürzere potentiell verbleibende Betriebszugehörigkeit, so dass Investitionen in betriebsspezifisches Humankapital weniger rentabel sind.

In der vierten Spezifikation in Tab. 5 nehmen wir zusätzlich eine binäre Variable für neu eingestellte Arbeiter auf. Deren Koeffizient hat zwar ein negatives Vorzeichen, ist aber nicht signifikant. In der fünften Spezifikation werden die Variablen zur Betriebszugehörigkeit ausgelassen. Unterschiede zu den vorherigen Spezifikationen sind vor allem beim Einfluss des Alters auszumachen, da dieses nun den Einfluss der Betriebszugehörigkeit absorbiert und einen signifikant positiven Einfluss hat. Der Eintrittsdummy hat nun ebenfalls einen signifikant negativen Einfluss. Aus diesem Ergebnis kann geschlossen werden, dass Neueingetretene großteils aufgrund fehlender betriebsspezifischer Qualifikationen, die außerhalb der Unternehmung nicht zu erlernen sind, auf unteren Ebenen eingestellt werden. Im Sinne der obigen Ausführungen kann dies dahingehend interpretiert werden, dass spezifisches Humankapital, welches im Laufe der betrieblichen Karriere „on the job“ erworben wird, eine besondere Rolle spielt. Auch wenn in den meisten 


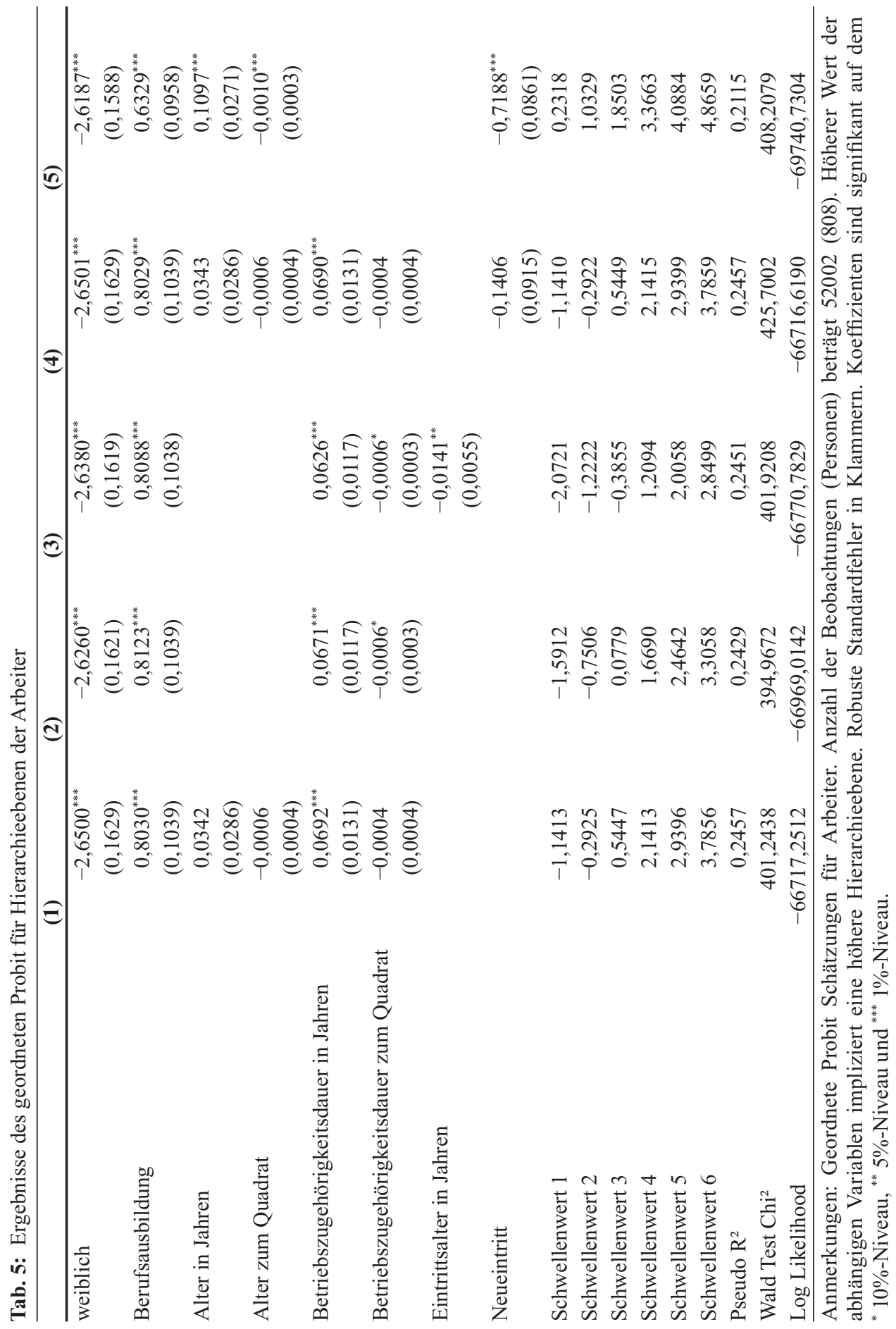


Publikationen eher Beförderungswahrscheinlichkeiten untersucht werden als direkt auf die Besetzung der Hierarchieebenen einzugehen, zeigt sich doch, dass in beiden Untersuchungsgegenständen vergleichbare Variablen eine Rolle spielen. So zeigt sich bspw. auch in Brüderl (1991), dass Männer eine höhere Beförderungswahrscheinlichkeit als Frauen haben. Dies stimmt mit unserem Ergebnis überein, dass Frauen auf niedrigeren Ebenen tätig sind. In Chan (2006) zeigt sich ferner, dass die Beförderungswahrscheinlichkeit mit der Betriebszugehörigkeit ansteigt; allerdings mit einer abnehmenden Rate. Dies ist vergleichbar mit dem uns vorliegenden Ergebnis, dass die Wahrscheinlichkeit für Arbeiter mit der Betriebszugehörigkeit steigt auf einer höheren Ebene tätig zu sein. Überdies weißt diese Wahrscheinlichkeit auch bei uns eine abnehmende Tendenz auf.

In den Tab. 6 und 7 sind die Ergebnisse für technische und kaufmännische Angestellte enthalten, die sich nur geringfügig zwischen den beiden Bereichen unterscheiden. Auch hier zeigen sich signifikant negative Effekte für weibliche Angestellte und signifikant positive Effekte für die Bildungsabschlüsse. Im Gegensatz zu den Arbeitern hat aber das Alter der Beschäftigten einen signifikant positiven Einfluss, während die Betriebszugehörigkeit keinen signifikanten Einfluss hat. Lediglich in den Schätzungen ohne Alter hat die Dauer der Betriebszugehörigkeit einen signifikanten Einfluss, der aber eher der Messung des Alterseffekts in der Betriebszugehörigkeit zugerechnet werden muss. Das Eintrittsalter hat ebenfalls einen signifikanten Einfluss, der aber im Gegensatz zu Arbeitern positiv ist. Dies deutet darauf hin, dass Angestellte ihr vor dem Eintritt in das Unternehmen erworbenes allgemeines Humankapital produktiv einsetzen können. Dementsprechend fällt auch der Nachteil Neueingestellter bei technischen und kaufmännischen Angestellten geringer aus als bei Arbeitern. Insgesamt lassen die Ergebnisse den Schluss zu, dass die Qualifikationen der Angestellten zu einem hohen Grad allgemein sind. Obwohl die Ergebnisse für Angestellte zeigen, dass der Einfluss der Länge der Betriebszugehörigkeit gering ist, schließt dies die Existenz von betrieblichen Karrieren nicht aus, da auch Angestellte im Laufe ihrer Karriere höhere Ebenen besetzen. Aber im Unterschied zu Arbeitern haben Angestellte eher die Möglichkeit durch Betriebswechsel ihre Karriere zu fördern.

Die Schätzungen für Arbeiter und Angestellte ergaben einen signifikant negativen Effekt für Frauen, d.h. Frauen haben eine deutlich geringere Wahrscheinlichkeit auf höheren Ebenen tätig zu sein als Männer. Dieser Befund ist in der Literatur dokumentiert und kann verschiedenen Faktoren zugeschrieben werden (vgl. Cain, 1986; Altonji/ Blank, 1999; Pfeifer/Sohr, 2008). Eine mögliche Erklärung ist Diskriminierung. Da es sich um ein produzierendes Unternehmen handelt, können wir Diskriminierung durch Kunden, die lieber von Männern auf höheren Ebenen bedient werden wollen, weitgehend ausschließen. Jedoch besteht auch hier die Möglichkeit der Diskriminierung durch industrielle Großkunden, die aber in einem Wettbewerbsmarkt eher unwahrscheinlich ist. Zudem würde diese Art der Diskriminierung nicht die Arbeiter betreffen. Auch die Diskriminierung durch das Unternehmen selbst ist eher unwahrscheinlich, da es sich im globalen Wettbewerb befindet. So bleibt noch die Diskriminierung durch andere Arbeitnehmer, insbesondere durch männliche Vorgesetzte, die über Beförderungen und somit über den Aufstieg in der betrieblichen Hierarchie entscheiden. Aber auch hier ist nicht davon auszugehen, dass ein solches Verhalten auf Dauer geduldet würde. Es sei nur an die starke Position des Betriebsrates erinnert und daran, dass in der Personalabteilung, die in gewissen Grenzen auch Einfluss auf die Ausgestaltung und Besetzung der Hierarchie hat, 


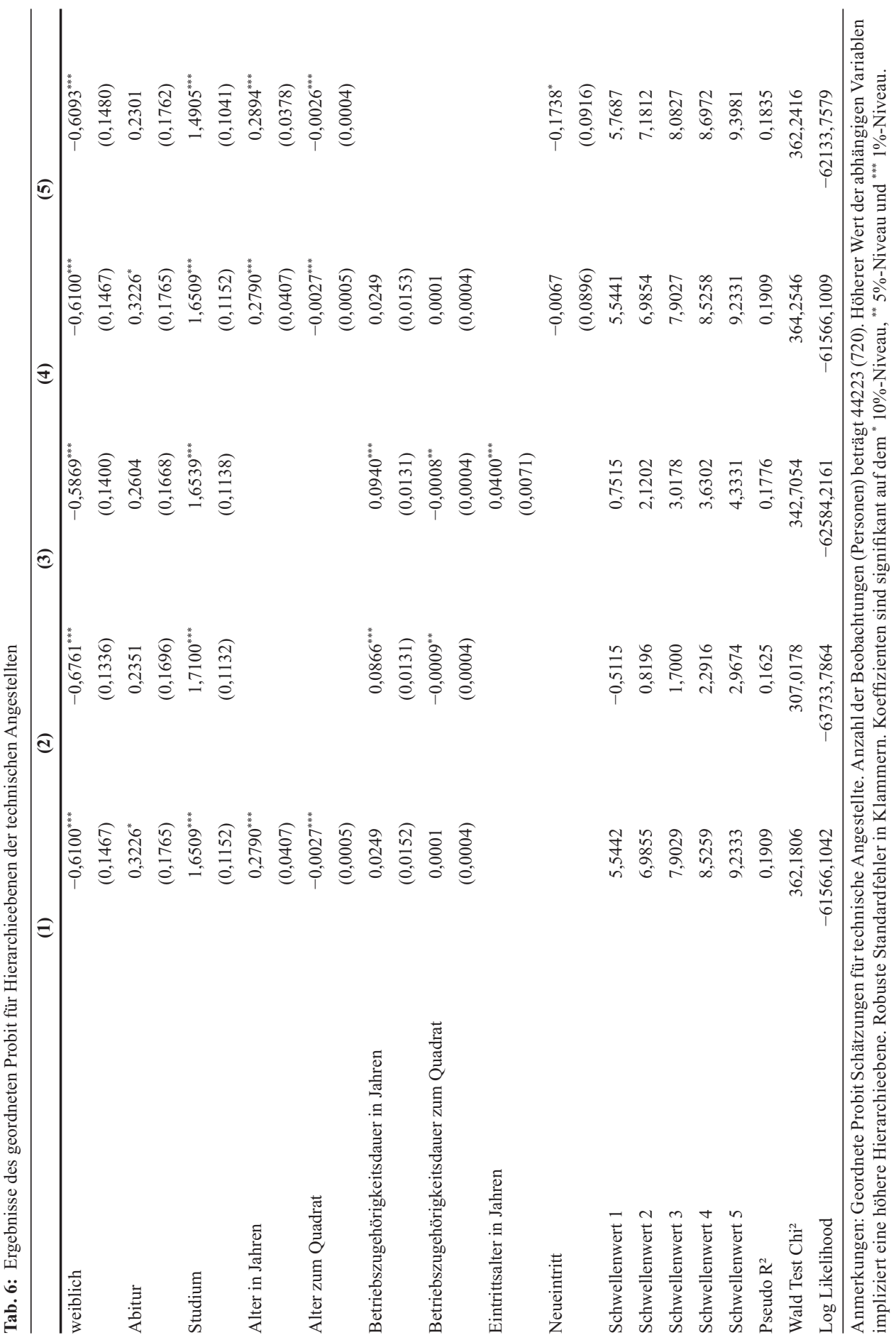




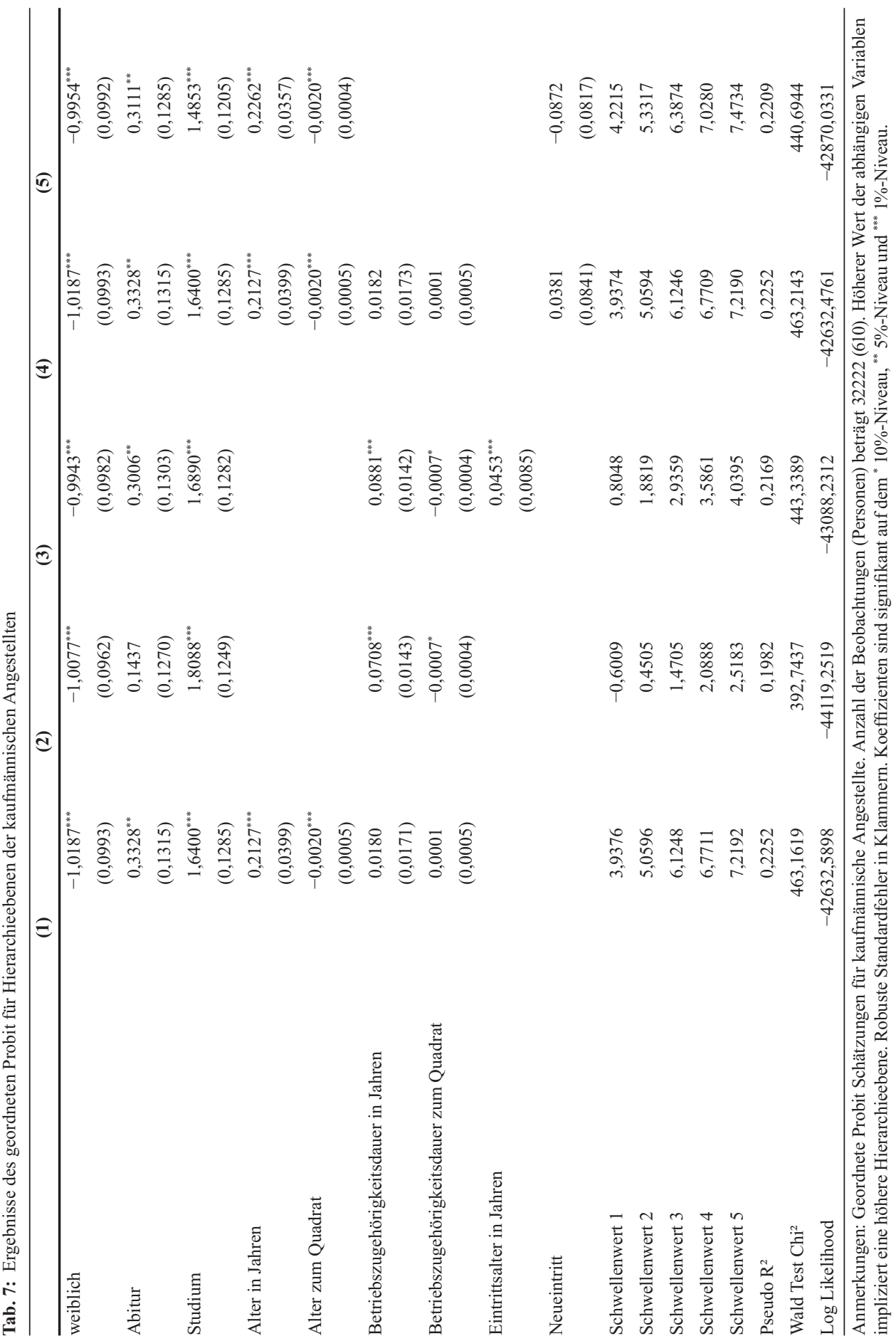


überwiegend Frauen beschäftigt sind. Eine alternative Erklärung ist eine geringere Produktivität von Frauen. Diese muss nicht zwangsläufig gegeben sein, sondern kann sich durch verschiedene Faktoren ergeben. Beispielsweise haben Frauen im Durchschnitt eine kürzere Dauer der Betriebszugehörigkeit, kündigen häufiger freiwillig, arbeiten weniger Stunden und sind häufiger krankheits- oder familienbedingt abwesend, so dass die Firma eher in das Humankapital und die betriebliche Karriere von Männern investiert (statistische Diskriminierung). Dies würde auch erklären, warum der Nachteil für Frauen bei Arbeitern größer als bei Angestellten ist.

In Tab. 8 und 9 wurden die geschätzten Wahrscheinlichkeiten aus den Modellen für männliche Beschäftigte auf den einzelnen Ebenen aller Bereiche in Abhängigkeit des Alters aufgeführt. Zur besseren Vergleichbarkeit liegt diesen Ergebnissen die jeweils fünfte Spezifikation zu Grunde. In den Spalten, die mit „Wahrscheinlichkeitsintervall“" bezeichnet sind, wurde der Wertebereich der einzelnen Wahrscheinlichkeiten auf der jeweiligen Ebene abgetragen. „Alter im Minimum“ und „Alter im Maximum“ geben dabei jeweils an, bei welchem Alter der geringste bzw. der höchste Wahrscheinlichkeitswert erreicht wird.

Für die Arbeiter (vgl. Tab. 8) fällt auf, dass sich die Wahrscheinlichkeit in der vierten Ebene zu arbeiten für Ausgebildete und nicht Ausgebildete auf einem sehr hohen Niveau befindet. Der Wertebereich bleibt für beide Gruppen über das gesamte Arbeitsleben relativ eng, wobei die Wahrscheinlichkeit in dieser Ebene tätig zu sein bei den nicht Ausgebildeten steigt und bei den Ausgebildeten zu Gunsten der höheren Ebenen sinkt. Die Wahrscheinlichkeit in einer höheren Ebene tätig zu sein, bleibt in beiden Gruppen jedoch unterhalb des Niveaus der vierten Ebene. Dies könnte dahingehend interpretiert werden, dass sich für die meisten Arbeiter ein Großteil des Arbeitslebens auf der vierten Ebene abspielt. Die Chancen aufzusteigen sind also vergleichsweise gering. Es ist allerdings zu beachten, dass die Wahrscheinlichkeitsintervalle für ausgebildete Arbeiter auf den Ebenen über 4 größer werden. So ist die Wahrscheinlichkeit für einen ausgebildeten Arbeiter mit 55 Jahren auf Ebene 7 tätig zu sein 14 mal höher als im Alter von 20 Jahren. Dieses Ergebnis bestätigt, dass betriebsinterne Karrieren insbesondere für Facharbeiter eine wesentliche Rolle spielen.

Tab. 8: Geschätzte Wahrscheinlichkeiten auf den einzelnen Ebenen für Arbeiter

\begin{tabular}{lllllll}
\hline & ohne Ausbildung & \multicolumn{9}{l}{ mit Ausbildung } & & \\
\hline & & \multicolumn{2}{l}{ Alter im } & \multicolumn{2}{l}{ Alter im } \\
\hline & $\begin{array}{l}\text { Wahrscheinlichkeitsin- } \\
\text { tervall }\end{array}$ & Min. & Max. & $\begin{array}{l}\text { Wahrscheinlichkeitsin- } \\
\text { tervall }\end{array}$ & Min. & Max. \\
\hline Ebene 1 & $0,0027-0,0591$ & 55 & 20 & $0,0003-0,0141$ & 55 & 20 \\
Ebene 2 & $0,0214-0,1642$ & 55 & 20 & $0,0042-0,0676$ & 55 & 20 \\
Ebene 3 & $0,0993-0,2991$ & 55 & 20 & $0,0321-0,2004$ & 55 & 20 \\
Ebene 4 & $0,4196-0,5515$ & 20 & 35 & $0,3550-0,5510$ & 55 & 23 \\
Ebene 5 & $0,0471-0,2202$ & 20 & 55 & $0,1255-0,2810$ & 20 & 55 \\
Ebene 6 & $0,0098-0,1085$ & 20 & 55 & $0,0409-0,2171$ & 20 & 55 \\
Ebene 7 & $0,0011-0,0316$ & 20 & 55 & $0,0074-0,1104$ & 20 & 55 \\
\hline
\end{tabular}

Anmerkung: Die Berechnungen basieren auf den Schätzergebnissen der fünften Spezifikation in Tab. 5. 


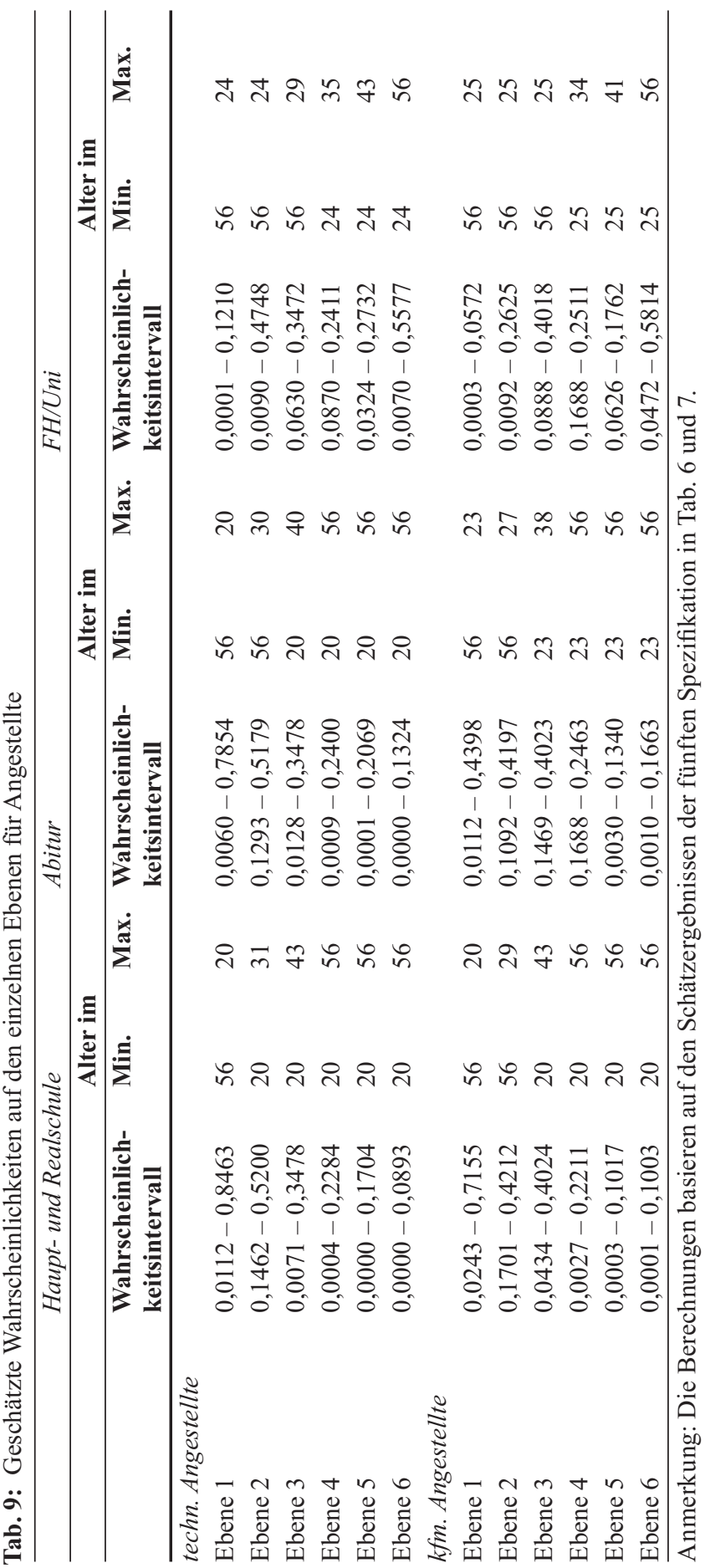




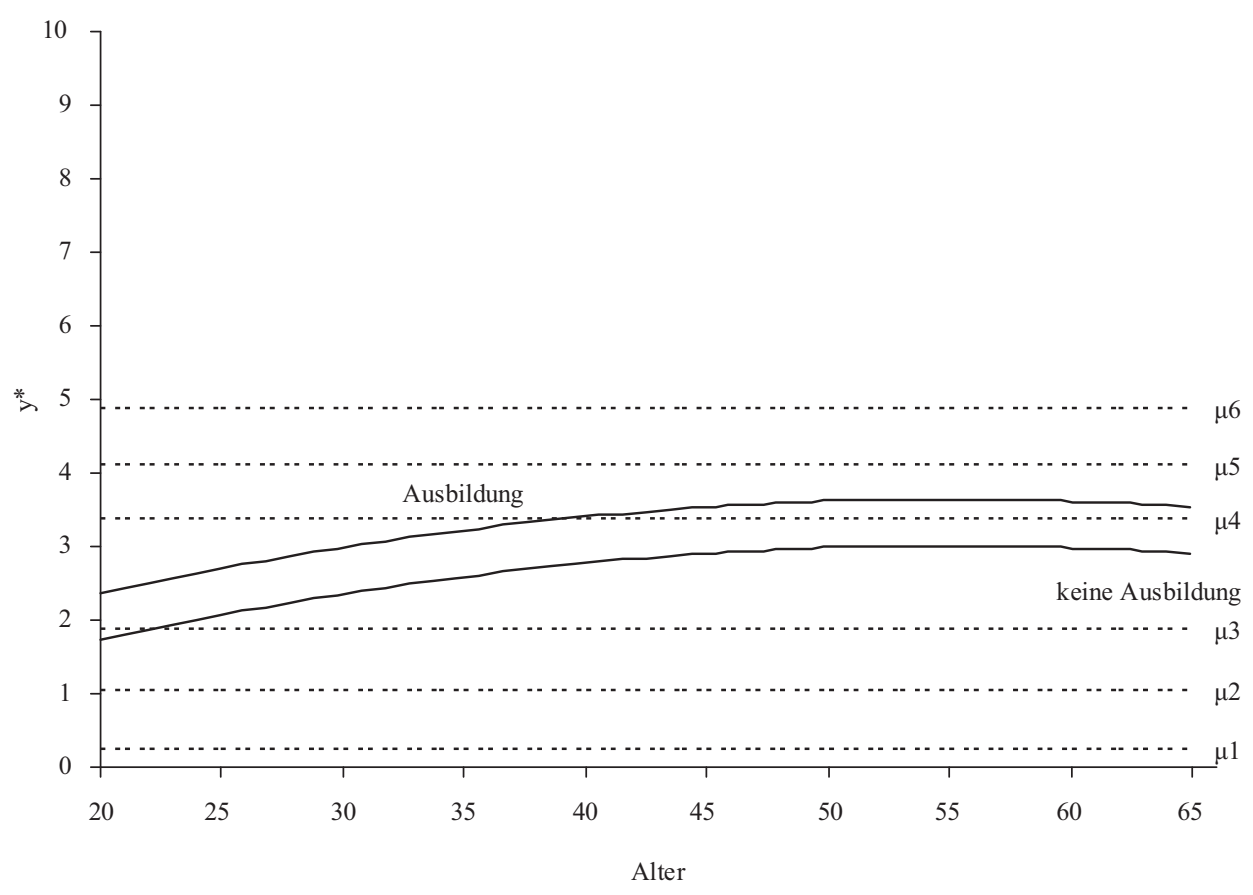

Abb. 1: Verteilungsverläufe für Arbeiter

Um einen besseren Überblick über den Verlauf der Wahrscheinlichkeiten zu bekommen wurden in Abb. 1 für männliche Arbeiter die $\hat{y}^{*}=x^{\prime} \hat{\beta}$ der fünften Spezifikation über dem Alter abgetragen. Die gestrichelten Linien stellen hierbei die Schwellenwerte dar. Die maximale Wahrscheinlichkeit in einer bestimmten Ebene tätig zu sein, ist immer dann erreicht, wenn sich ein Punkt auf den durchgezogenen Linien genau in der Mitte zwischen zwei Schwellenwerten befindet. Je weiter beide Schwellenwerte hierbei auseinander liegen, umso größer ist die Wahrscheinlichkeit in diesem Punkt. Rechts und links der Mitte zwischen zwei Schwellenwerten ist die Wahrscheinlichkeit allerdings kleiner. In Abb. 1 ist gut zu erkennen, dass die Schwellenwerte über- und unterhalb der vierten Ebene, also außerhalb des Bereiches zwischen $\mu_{3}$ und $\mu_{4}$, verhältnismäßig eng beieinander liegen. Dass die Wahrscheinlichkeiten außerhalb der vierten Ebene tätig zu sein relativ klein sind, wird daran deutlich, dass die durchgezogenen Linien die Ebenen 7 und 6 sowie 1, 2 und 3 nicht durchqueren.

Betrachtet man die Ergebnisse für die Angestellten (vgl. Tab. 9), fällt auf, dass die Wertebereiche auf den einzelnen Ebenen sehr viel größere Intervalle umfassen. Somit sind die Positionen in der jeweiligen Hierarchie stark altersabhängig. Dies wird auch in den Abb. 2 und 3 deutlich. Die Verläufe der durchgezogenen Linien sind vergleichsweise steiler und die Schwellenwerte liegen sehr nah beieinander. Ein derartiges Ergebnis deutet darauf hin, dass die Berufserfahrung nach Abschluss der formalen Bildung eine besondere Rolle spielt. Da die Betriebszugehörigkeit bei den Angestellten keine entscheidende Rolle spielt, ist davon auszugehen, dass für die Angestellten des betrachteten Unternehmens insbeson- 


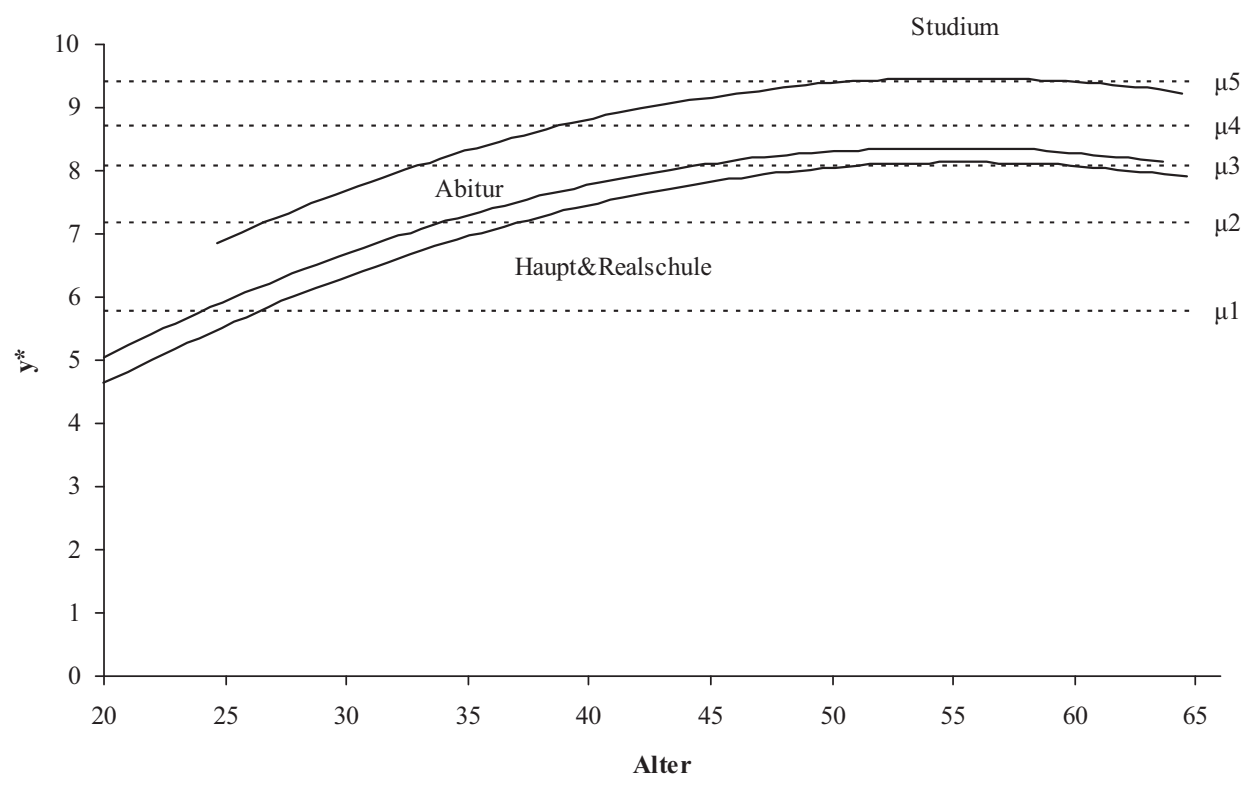

Abb. 2: Verteilungsverläufe für technische Angestellte

dere die Akkumulation von allgemeinem Humankapital nach der formalen Ausbildung wichtig ist. Diese Vermutung wird dadurch gestützt, dass für relativ junge Hochschulabgänger sowohl bei den technischen als auch bei den kaufmännischen Angestellten die Wahrscheinlichkeit recht hoch ist, sogar unter der dritten Ebene eingeordnet zu werden. Mit steigendem Alter wird diese Wahrscheinlichkeit jedoch verschwindend gering.

Weiterhin fällt auf, dass die Wahrscheinlichkeitsintervalle der Universitätsabsolventen auf den oberen Rängen deutlich über denen der anderen Angestellten liegen und auf den unteren Rängen deutlich darunter. Beispielsweise ist die Wahrscheinlichkeit einen außertariflich bezahlten Posten zu bekleiden für einen 56-jährigen Akademiker ca. 5,7-mal größer als für einen gleichaltrigen Haupt- oder Realschüler. Dies macht eine starke Segmentierung der Beschäftigten in Bildungsbereiche deutlich, die sich im Karriereverlauf verstärkt. Bemerkenswert ist außerdem, dass die Wahrscheinlichkeit für Akademiker beider Bereiche in einer außertariflichen Stelle tätig zu sein, innerhalb der Karriere von einem Prozent auf über 50 Prozent ansteigt. Für die Angestellten mit einem Hochschulabschluss fällt sowohl das Wahrscheinlichkeitsniveau als auch der altersbedingte Anstieg der Wahrscheinlichkeit auf der höchsten Ebenen tätig zu sein wesentlich größer aus als für die anderen Bildungsgruppen. Dieses Ergebnis bestätigt, dass formale Bildungsabschlüsse eine wesentliche Determinante der Karriere von Angestellten darstellen und zeigt auf, dass bei Angestellten allgemeines Humankapital eine besondere Rolle spielt. Durch diese Tatsache lässt sich erklären, warum der Arbeitsmarkt der Angestellten wesentlich stärker gegenüber dem externen Markt geöffnet ist. 


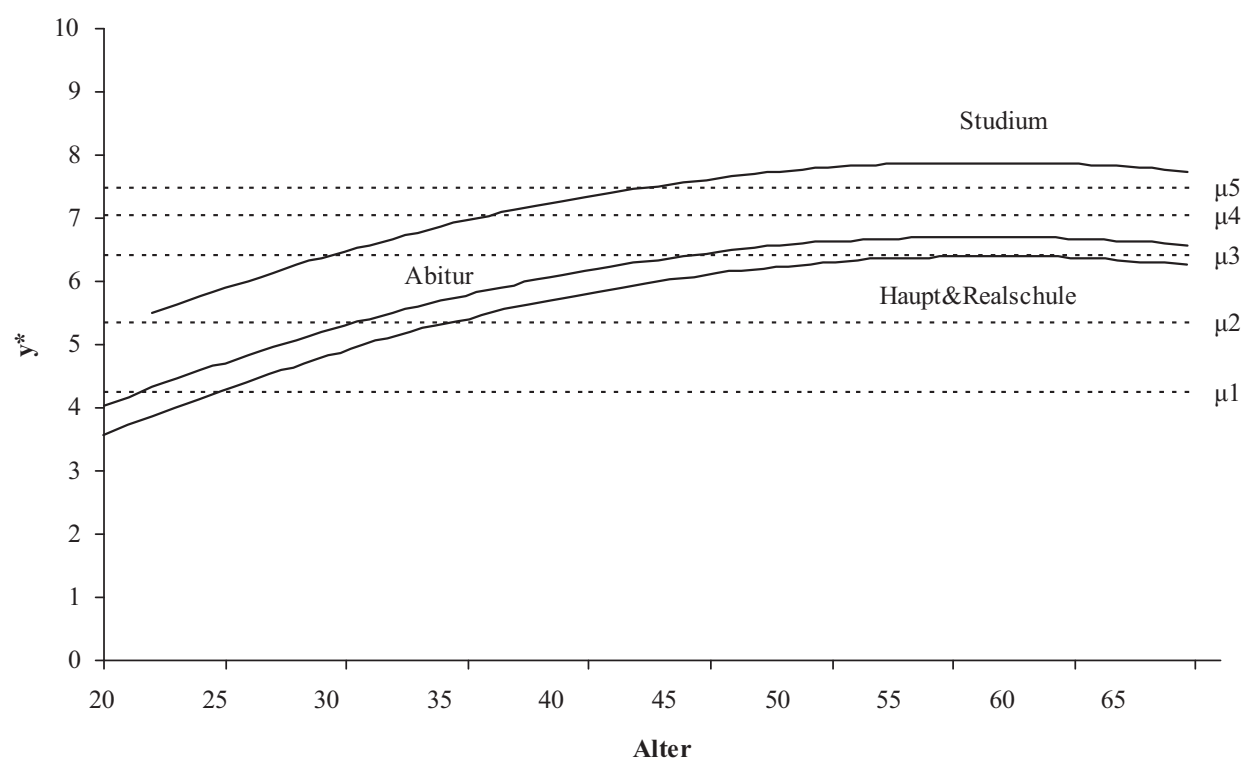

Abb. 3: Verteilungsverläufe für kaufmännische Angestellte

\section{Zusammenfassung und Diskussion}

Auch wenn sowohl für Angestellte als auch für Arbeiter - und insbesondere für Facharbeiter - des betrachteten Unternehmens betriebsinterne Karrieren eine wichtige Rolle spielen, zeigt unsere Untersuchung, dass der Arbeitsmarkt für Arbeiter wesentlich stärker vom externen Markt isoliert ist als der Arbeitsmarkt für Angestellte. Weiterhin wurde gezeigt, dass dieses Ergebnis, der Theorie von Doeringer und Piore (1971) entsprechend, mit der verstärkten Existenz von spezifischem Humankapital in Zusammenhang mit „on the job" Training einhergeht. Ferner wird die Position in der Hierarchie, insbesondere bei den Angestellten, maßgeblich von der formalen Schul- und Berufsausbildung der Beschäftigten determiniert. Obwohl die einzelnen hierarchischen Ebenen bzw. Lohn- und Gehaltsgruppen generell für alle Beschäftigten zugänglich zu sein scheinen, ist doch eine deutliche Segmentierung der Beschäftigten im Sinne der Richtlinien des Tarifvertrages auszumachen. Die Unterschiede zwischen den Bildungsgruppen werden im Karriereverlauf darüber hinaus deutlich verstärkt.

In Anbetracht des letzten Ergebnisses soll hier noch einmal auf die Besonderheiten des deutschen Arbeitsmarktes eingegangen werden. Zum einen werden hierzulande die Beschäftigten aufgrund des dreigliedrigen Schulsystems, der dualen Ausbildung und der Hochschulpolitik schon sehr früh in bestimmte Bildungsgruppen untergliedert. Zum anderen werden die Lohn- und Gehaltsgruppen, nach denen sich die hierarchische Struktur vieler Unternehmen richtet, im Rahmen eines Flächentarifs für die gesamte Branche ausgehandelt und bleiben vielfach über einen langen Zeitraum stabil (vgl. Bahnmüller et al., 1999). In Tarifverträgen wird neben den grob definierten Tätigkeiten vielfach explizit auf die einzelnen Abschlüsse des deutschen Bildungssystems Bezug genommen. In 
unseren Ergebnissen zeigt sich allerdings, dass in keinem der Bereiche eine vollständige Segmentierung nach Bildungsabschlüssen zu erkennen ist. Die Richtlinien des Tarifvertrages werden in diesem Sinne von der Unternehmung nicht als zwingend betrachtet und sind sowohl nach unten als auch nach oben hin offen. Es ist daher fraglich, ob das deutsche Schul- und Ausbildungssystem die potentiellen Beschäftigten tatsächlich mit so aussagekräftigen Signalen ausstattet, dass diese dann von den Unternehmen perfekt genutzt werden können. Außerdem bleibt offen, inwieweit die vorgefundene Segmentierung innerhalb des Unternehmens ökonomisch rational ist und ob die Beschäftigten anders auf ihre Stellen verteilt würden, wenn es keinen tarifvertraglichen Rahmen geben würde. Abschließend ist anzumerken, dass es sich bei unserer Arbeit nur um eine Fallstudie handelt und die Ergebnisse nicht uneingeschränkt verallgemeinert werden können.

\section{Anmerkungen}

1 In dieser Arbeit werden die Hierarchien vor allem unter dem Allokationsaspekt betrachtet. $\mathrm{Zu}$ hierarchischen Strukturen als Anreizinstrument vgl. Lazear und Rosen (1981) sowie Lazear (1992).

2 Von der Personalabteilung wurde uns bestätigt, dass unter Beförderungen Versetzungen von einer Lohn- und Gehaltsgruppe in die nächste verstanden werden. Versetzungen von einem Job in einen anderen innerhalb einer Gruppe werden hingegen nicht unter dem Begriff einer Beförderung geführt. Da sich die Einstufung nach Schulabschlüssen und Tätigkeiten richtet, sind somit die Voraussetzungen erfüllt, um eine hierarchische Struktur abzubilden. Informationen über genauere Funktionen der Arbeitnehmer, die eine feinere Untergliederung der Hierarchie erlauben würde, sind leider nicht im Datensatz verfügbar.

3 Eine Konstante wurde nicht eingeführt, da diese bei der Schätzung nicht von den Schwellenwerten zu trennen ist. Man könnte aber beispielsweise den ersten Schwellenwert als konstanten Term bezeichnen. 


\section{Anhang}

Tab. A. 1: Variablen für multivariate Schätzungen

\begin{tabular}{|c|c|c|c|c|}
\hline & Mittelwert & $\begin{array}{l}\text { Standardab- } \\
\text { weichung }\end{array}$ & Minimum & Maximum \\
\hline \multicolumn{5}{|l|}{ Arbeiter } \\
\hline Berufsausbildung & 0,7253 & 0,4463 & 0,00 & 1,00 \\
\hline Alter in Jahren & 42,3108 & 8,8021 & 19,20 & 65,08 \\
\hline Betriebszugehörigkeitsdauer in Jahren & 15,1300 & 9,1296 & 0,01 & 49,11 \\
\hline Eintrittsalter in Jahren & 27,1808 & 7,5714 & 13,70 & 55,53 \\
\hline weiblich & 0,2011 & 0,4008 & 0,00 & 1,00 \\
\hline Neueintritt & 0,0016 & 0,0404 & 0,00 & 1,00 \\
\hline Hierarchieebene & 4,0192 & 1,4931 & 1,00 & 7,00 \\
\hline Hierarchieebene 1 & 0,0908 & 0,2873 & 0,00 & 1,00 \\
\hline Hierarchieebene 2 & 0,0715 & 0,2577 & 0,00 & 1,00 \\
\hline Hierarchieebene 3 & 0,1033 & 0,3044 & 0,00 & 1,00 \\
\hline Hierarchieebene 4 & 0,3949 & 0,4888 & 0,00 & 1,00 \\
\hline Hierarchieebene 5 & 0,1852 & 0,3885 & 0,00 & 1,00 \\
\hline Hierarchieebene 6 & 0,1101 & 0,3130 & 0,00 & 1,00 \\
\hline Hierarchieebene 7 & 0,0442 & 0,2055 & 0,00 & 1,00 \\
\hline \multicolumn{5}{|l|}{ technische Angestellte } \\
\hline Haupt- oder Realschule & 0,5334 & 0,4989 & 0,00 & 1,00 \\
\hline Abitur & 0,0978 & 0,2970 & 0,00 & 1,00 \\
\hline Studium & 0,3688 & 0,4825 & 0,00 & 1,00 \\
\hline Alter in Jahren & 43,7970 & 9,2179 & 18,19 & 65,98 \\
\hline Betriebszugehörigkeitsdauer in Jahren & 14,6415 & 9,4245 & 0,01 & 48,20 \\
\hline Eintrittsalter in Jahren & 29,1554 & 7,0070 & 13,70 & 55,36 \\
\hline weiblich & 0,1443 & 0,3514 & 0,00 & 1,00 \\
\hline Neueintritt & 0,0040 & 0,0628 & 0,00 & 1,00 \\
\hline Hierarchieebene & 3,5315 & 1,5166 & 1,00 & 6,00 \\
\hline Hierarchieebene 1 & 0,0664 & 0,2490 & 0,00 & 1,00 \\
\hline Hierarchieebene 2 & 0,2321 & 0,4222 & 0,00 & 1,00 \\
\hline Hierarchieebene 3 & 0,2492 & 0,4326 & 0,00 & 1,00 \\
\hline Hierarchieebene 4 & 0,1582 & 0,3649 & 0,00 & 1,00 \\
\hline Hierarchieebene 5 & 0,1440 & 0,3511 & 0,00 & 1,00 \\
\hline Hierarchieebene 6 & 0,1500 & 0,3571 & 0,00 & 1,00 \\
\hline \multicolumn{5}{|l|}{ kaufmännische Angestellte } \\
\hline Haupt- oder Realschule & 0,4166 & 0,4930 & 0,00 & 1,00 \\
\hline Abitur & 0,2573 & 0,4372 & 0,00 & 1,00 \\
\hline Studium & 0,3261 & 0,4688 & 0,00 & 1,00 \\
\hline Alter in Jahren & 39,9688 & 9,4259 & 18,66 & 65,09 \\
\hline Betriebszugehörigkeitsdauer in Jahren & 12,7122 & 9,7491 & 0,02 & 45,03 \\
\hline Eintrittsalter in Jahren & 27,2566 & 7,6965 & 15,51 & 62,26 \\
\hline weiblich & 0,4818 & 0,4997 & 0,00 & 1,00 \\
\hline
\end{tabular}


Tab. A. 1: Fortsetzung von S. 660

\begin{tabular}{lllll}
\hline & Mittelwert & $\begin{array}{l}\text { Standardab- } \\
\text { weichung }\end{array}$ & Minimum & Maximum \\
\hline Neueintritt & 0,0060 & 0,0772 & 0,00 & 1,00 \\
Hierarchieebene & 3,1149 & 1,5782 & 1,00 & 6,00 \\
Hierarchieebene 1 & 0,1572 & 0,3640 & 0,00 & 1,00 \\
Hierarchieebene 2 & 0,2462 & 0,4308 & 0,00 & 1,00 \\
Hierarchieebene 3 & 0,2620 & 0,4397 & 0,00 & 1,00 \\
Hierarchieebene 4 & 0,1296 & 0,3359 & 0,00 & 1,00 \\
Hierarchieebene 5 & 0,0692 & 0,2539 & 0,00 & 1,00 \\
Hierarchieebene 6 & 0,1358 & 0,3426 & 0,00 & 1,00 \\
\hline
\end{tabular}

Anmerkung: Anzahl der Beobachtungen (Personen) beträgt bei Arbeitern 52002 (808), bei technischen Angestellten 44223 (720) und bei kaufmännischen Angestellten 32222 (610).

\section{Literatur}

Acosta P (2004) Promotions, State Dependence and Intrafirm Job Mobility: Evidence from Personnel Records, Unpublished Working Paper

Agrawal A, Knober CR, Tsoulouhas T (2006) Are Outsiders Handicapped in CEO Successions?. J Corp Finan 12:619-644

Altonji, JG, Blank RM (1999) Race and gender in the labor market. Handbook of Labor Economics 3:3143-3259

Bahnmüller R, Bispinck R, Weiler A (1999) Tarifpolitik und Lohnbildung in Deutschland am Beispiel ausgewählter Wirtschaftszweige, WSI- Diskussionspapier Nr. 79

Baker G, Gibbs M, Holmstrom B (1994a) The Internal Economics of the Firm: Evidence from Personnel Data. Quart J Econ 19(4):882-919

Baker G, Gibbs M, Holmstrom B (1994b) The Wage Policy of a Firm. Quart J Econ 19(4):921-955

Becker GS (1964) Human Capital: A Theoretical and Empirical Analysis. Columbia University Press, New York

Brüderl J (1991) Mobilitätsprozesse in Betrieben Sozialwissenschaftliche Arbeitsmarktforschung. Campus Verlag, Frankfurt am Main und New York

Cain GG (1986) The economic analysis of labor market discrimination: a survey. Handbook of Labor Economics 1:693-785

Chan W (1996) External Recruitment versus Internal Promotion. J Lab Econ 14(4):555-570

Chan W (2006) External Recruitment and Intrafirm Mobility. Econ Inquiry 44(1):169-184

Doeringer P, Piore M (1971) Internal Labor Markets and Manpower Analysis. D. C. Heath, Lexington, MA

Dohmen T, Kriechel B, Pfann G (2004) Monkey Bars and Ladders: The Importance of Lateral and Vertical Job Mobility in Internal Labor Market Careers. J Population Econ 17(2):198-228

Dunlop JT (1958) Industrial Relations Systems. Henry Holt \& Company Inc., New York

Dunlop JT (1988) How Labor Markets Work. Lexington Books, Lexington, MA

Gibbons RS, Waldman M (1999) A Theory of Wage and Promotion Dynamics Inside Firms. Quart J Econ 114(4):1321-1358

Gibbons RS, Waldman M (2006) Enriching a Theory of Wage and Promotion Dynamics inside Firms. J Lab Econ 24(1):59-107

Grund C Kräkel M (2001) Interne Arbeitsmärkte und betriebliche Entgeltstrukturen - eine empirische Untersuchung, ZfB, Ergänzungsheft 1/2001, S. 1-25 
Grund C (2002) Empirische Analyse auf Basis betrieblicher Personaldaten. WiSt 31(11):627-632

Kerr C (1954) The Balkanization of Labor Markets. In: Bakke EW (Hrsg) Labor Mobility and Economic Opportunity - Essays, John Wiley \& Sons, New York, S 92-110

Köhler C, Preisendörfer P, (1989) Betrieblicher Arbeitsmarkt im Umbruch: Analysen zur Mobilität, Segmentation und Dynamik in einem Großbetrieb. Campus Verlag, Frankfurt am Main und New York

Lazear E (1992) The Job as a Concept. In: William JB (Hrsg) Performance Measurement, Evaluation, and Incentives, MA: Harvard Business School Press, Boston, S. 183-215

Lazear E, Oyer P (2002) Ports of Entry, Unpublished Working Paper

Lazear E, Oyer P (2004a) The Structure of Wages and Internal Mobility. The American Economic Review 94(2):212-216

Lazear E, Oyer P (2004b) Internal and External Labour Markets: A Personnel Economics Approach. Lab Econ 11(5):527-554

Lazear E, Rosen S (1981) Rank-Order Tournaments as Optimum Labor Contracts. J Polit Economy 89(5):841-864

Lima F (2000) Internal Labor Markets: A Case Study, Unpublished Working Paper

Lin M-J (2005) Opening the Black Box: The Internal Labor Markets of Company X. Ind Relat 44(4):659-706

Oberst M, Schank T, Schnabel C (2007) Interne Arbeitsmärkte und Einsatz temporärer Arbeitsverhältnis: Eine Fallstudie mit Daten eines deutschen Dienstleistungsunternehmens. Z Betriebswirtschaft 77:1159-1177

Pfeifer C (2008) An empirical note on wages in an internal labour market. Econ Letters 99:570-573

Pfeifer C, Sohr T (2008) Analysing the Gender Wage Gap Using Personnel Records of a Large German Company, IZA Discussion Paper No. 3533

Wachter ML, Wright R (1990) The Economics of Internal Labor Markets. Ind Relat 29(2):240-262

\title{
Internal Labour Markets, Hierarchies and New Entrants: An Empirical Analysis with Personnel Records
}

\begin{abstract}
Many empirical studies which deal with internal labour markets are related to the work of Doeringer and Piore (1971). The majority of these studies cannot find closed internal labour markets with entry positions at the lower end of a firm's hierarchy. Most of these studies, however, use data from financial and service industries, managerial or other white-collar workers. Doeringer and Piore (1971) state that closed internal labour markets with clearly defined entry positions should be found especially for blue-collar workers in the manufacturing industry, because firm specific human capital is of large importance in these industries. In this paper, a large data set of a manufacturing company with blue-collar and white-collar workers is used. We show that the internal labour market of blue-collar workers is more closed than that of white-collar workers. Moreover, we find that firm specific human capital plays a major role in determining the hierarchical position of blue-collar workers. Entries of white-collar workers, however, appear at all hierarchical positions. Furthermore, formal education plays a major role in allocating the white-collar workers.
\end{abstract}

Keywords: Efficient allocation · Internal labour markets · Personnel records 